\title{
Direct Growth of Graphene at Low Temperature for Future Device Applications
}

\author{
Bum Jun Kim**, Tuqeer Nasir**, and Jae-Young Choi ${ }^{* * * *, \dagger}$ \\ *School of Advanced Materials Science \& Engineering, Sungkyunkwan University, Suwon 16419, Korea \\ **SKKU of Advanced Institute of Nanotechnology (SAINT), Sungkyunkwan University, Suwon 16419, Korea
}

(Received April 2, 2018; Revised May 13, 2018; Accepted May 14, 2018)

\begin{abstract}
The development of two-dimensional graphene layers has recently attracted considerable attention because of its tremendous application in various research fields. Semi-metal materials have received significant attention because of their excellent biocompatibility as well as distinct physical, chemical, and mechanical properties. Taking into account the technical importance of graphene in various fields, such as complementary metal-oxide-semiconductor technology, energy-harvesting and -storage devices, biotechnology, electronics, light-emitting diodes, and wearable and flexible applications, it is considered to be a multifunctional component. In this regard, material scientists and researchers have primarily focused on two typical problems: i) direct growth and ii) low-temperature growth of graphene. In this review, we have considered only cold growth of graphene. The review is divided into five sections. Sections 1 and 2 explain the typical characteristics of graphene with a short history and the growth methods adopted, respectively. Graphene's direct growth at low temperatures on a required substrate with a well-established application is then precisely discussed in Sections 3 and 4. Finally, a summary of the review along with future challenges is described in Section 5.
\end{abstract}

Key words : Direct growth of graphene, Low-temperature growth of graphene, Chemical vapor deposition, Physical \& chemical properties, Multifunctional applications

\section{Introduction}

$\mathrm{G}$ raphene is a single-layer two-dimensional (2D) form of Tgraphite and exhibits semi-metallic properties. The unique physical, chemical, and mechanical properties of graphene have attracted tremendous research interest from the leading scientists of the world. ${ }^{1-3)}$ Graphene has a hexagonal honeycomb crystal structure. A single hexagonal structure of graphene consists of six carbon atoms, which form three strong $\sigma$-bonds with three adjacent atoms by hybridization of the $s, p_{x}$, and $p_{y}$ atomic orbitals and $\pi-\pi^{*}$ bonds by overlapping of the $p_{z}$ orbital with the next carbon atom in the hexagon. The conduction band and valance band of graphene are symmetrical at the Dirac point with Fermi surface pins at the intersection points (overlapping points of $\pi-\pi$ and $\left.\pi^{*}-\pi^{*}\right)$. This phenomenon enables graphene to behave like a metal with a vanishing Fermi surface or a semiconductor having zero bandgap. ${ }^{4)}$ Further, graphene can form different structures due to its wrappable nature because of the presence of zero-dimensional fullerene structures (Buckyball), one-dimensional carbon nanotubes (rolled layers of graphene that can be single- or multilayered), two dimensional graphene sheets and three-dimensional (3D) stacked layers of graphite. These properties have enabled

\footnotetext{
${ }^{\dagger}$ Corresponding author: Jae-Young Choi

E-mail : jy.choi@skku.edu

Tel : +82-31-290-7353 Fax : +82-31-290-7410
}

graphene to be considered as a very strong candidate for wide applications in the fields of nanoscience and nanotechnology, including electronics, optoelectronics, energy devices, capacitors, sensors, transparent conductors, bio-devices and wearable devices. ${ }^{5-9)}$ In addition, graphene has a unique crystallographic structure that is appropriately modifiable, making it a potential candidate for nanomedicine and biotechnology applications such as drug delivery systems, bioimaging, biosensing, antimicrobial applications, tissue engineering, and cell culturing.

The synthesis of graphene was first achieved using micromechanical cleavage and epitaxial growth on silicon carbide at elevated temperatures. Further, a significant improvement in the productivity of graphene was obtained by the exfoliation of graphene in organic solvents and water solutions. To utilize graphene to its maximum potential in practical applications, it is very crucial to produce high-quality (i.e., defect-free) structures by adopting simple, scalable, and economical techniques employing easy processable methodology. In this regard, researchers throughout the world have adopted various top-down and bottom-up methods such as mechanical exfoliation, carbon nanotube unzipping, epitaxial growth, self-assembly of surfactants, chemical vapor deposition (CVD), and chemical conversion to realize graphene structures on different substrates, thus achieving good structure quality with negligible or no defects. ${ }^{10-25)}$ To date, various strategies have been adopted to obtain graphene monolayers, including CVD, mechanical and liq- 
uid-based exfoliation of graphene flakes from graphite, ${ }^{26-28)}$ epitaxial growth using crystal substrates ${ }^{29-32)}$ and graphene oxide reduction using solution-based processes. ${ }^{33-38)}$ The remarkable advancement of CVD has facilitated the fabrication of large-scale graphene monolayers on different substrates. $^{3946)}$ As a result, graphene monolayers can be feasibly utilized for different applications, especially in complementary metal-oxide-semiconductor (CMOS) technology. These versatile synthesis techniques have made it possible to use single-layer graphene (SLG) in CMOS technology for other applications. ${ }^{47)}$

SLG has been recently developed on metal substrates using catalytic CVD. This has facilitated the development of versatile graphene-based devices and applications. ${ }^{39-46,48)}$ However, graphene monolayers grown on metal substrates using CVD cannot be used directly used for electronic applications and require an additional step of graphene transfer to an appropriate substrate. Several approaches have been developed to transfer graphene on required substrates, such as wet transfer/etching, dry transfer/exfoliation, ${ }^{26-28)}$ electrochemical delamination, ${ }^{50-52)}$ and bubbling transfer. ${ }^{49)}$ However, these methods degrade/damage the quality of the transferred graphene to some extent. ${ }^{53)}$ Therefore, graphene transfer is inconvenient and results in the generation of several mechanical and chemical defects, thus limiting the efficiency of graphene-based electronic devices. ${ }^{54}$ Keeping this in mind, the development of a more direct and facile method for graphene growth on desired substrates is of great importance to achieve efficient and high-quality devices. Moreover, several intended applications of graphene require it to be conformally grown on 3D surfaces, such as microoptics, ${ }^{55)}$ silicon solar cells, ${ }^{56)}$ pressure sensors, ${ }^{57)}$ microelectromechanical systems ${ }^{58)}$ and CMOS technology. ${ }^{47)}$ Therefore, researchers have investigated numerous direct growth systems based on the efficient utilization of the intrinsic properties of CVD-grown graphene. However, the temperature commonly used for graphene growth is considerably high $\left(\sim 1,000^{\circ} \mathrm{C}\right)$, which can deteriorate the dielectric layer, semiconducting layer and conducting layer in the device. Therefore, the combination of two important techniques, direct growth and low-temperature growth, is required for the practical application of graphene in future devices. In addition, the direct growth of graphene at low temperatures is desirable for the reduction in the overall production cost by i) avoiding graphene transfer, which involves the use of numerous chemicals and a sacrificial substrate, ii) decreasing the number of operations to enhance energy efficiency, and iii) avoiding the need to store and transport delicate graphene samples.

\section{Processes Adopted for Graphene Growth}

Over the last decade, high-quality graphene has been mostly synthesized using thermal CVD and plasmaenhanced CVD (PECVD) at low temperatures by adopting different hydrocarbon precursors and metal catalysts. Tran- sition metals have been used as catalysts or sacrificial layers along with several carbon precursors to achieve highquality graphene layers over large areas. ${ }^{59)}$ The transition metal substrate in this system acts as a catalyst to lower the energy barrier of the reaction and drive graphene growth. Graphene is mostly grown using methane as the carbon precursor and high-quality SLG is achieved at temperatures of $\sim 1000^{\circ} \mathrm{C}^{41,60,61)}$ To overcome the issues related to high-temperature graphene growth using methane, alternative solid/ liquid hydrocarbon precursors that can be decomposed at lower temperatures have attracted considerable attention. Thus, the temperature required for graphene growth in thermal CVD and PECVD has greatly decreased to around $300^{\circ} \mathrm{C}$ due to the use of different carbon sources such as ethylene, benzene, ethanol, acetylene, and toluene along with other solid hydrocarbons. ${ }^{62-66)}$

The commercialization of graphene-based devices is limited by many factors, among which high reaction temperatures during the growth process and the need to transfer of graphene to appropriate substrates are the crucial ones, as CMOS technology and flexible-device processing require operating temperatures below $600^{\circ} \mathrm{C}$. Hence, low-temperature graphene growth without compromising on graphene properties is highly desirable. In this regard, this review focuses on the important breakthroughs and studies on lowtemperature and direct growth of graphene and covers the physical properties of graphene along with its applications. Here, we have focused on the synthesis of SLG by low-temperature and direct growth using different CVD techniques and have analyzed its electrical and optical properties along with crystalline quality of the synthesized graphene. Finally, recent developments in graphene growth are summarized and future perspectives for commercialization along with related challenges are discussed. Thus, we believe that this review on low-temperature and direct growth of graphene is timely and provides an overview of the methods and technologies adopted to achieve high-quality graphene for application in devices.

\section{Direct Growth on Suitable Substrates}

To avoid the issues related to graphene transfer, two different growth approaches, catalytic growth and non-catalytic growth, have been used for the growth of graphene monolayers directly on the desired substrates. ${ }^{67-69)}$ Graphene monolayers have been grown successfully on flexible and rigid substrates using catalytic and non-catalytic growth processes ${ }^{55,67-100)}$ which are briefly described in the following sections.

\subsection{Catalytic growth}

Direct growth of SLG on quartz, sapphire, silicon, silicon dioxide, fused silica, and hexagonal boron nitride substrates using a metal catalyst (copper, nickel, tungsten, etc.) has been thoroughly investigated. ${ }^{78,82-84,86,89,91-93,95,101)}$ In this process, the chemical reaction involved in graphene growth, 

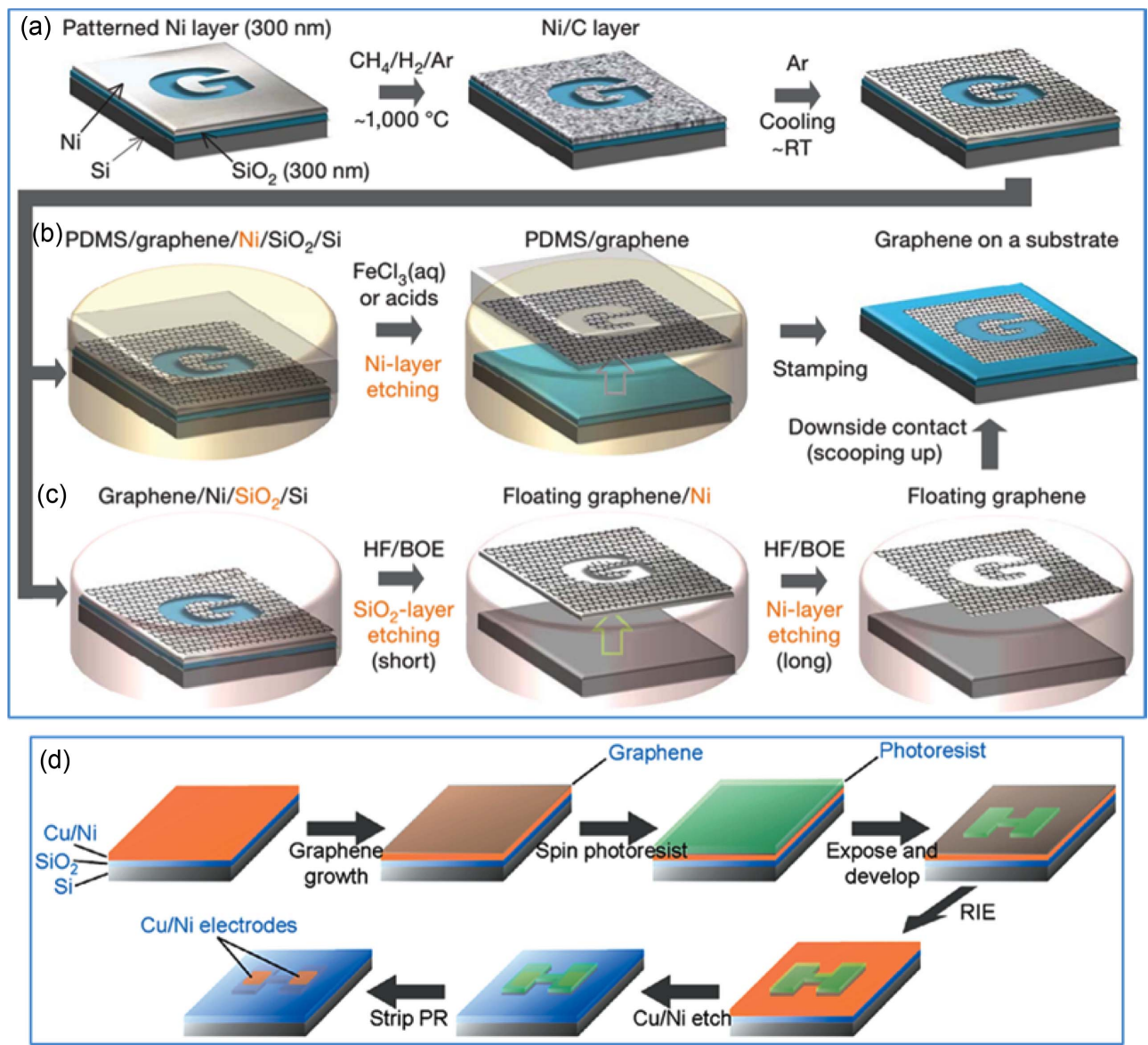

Fig. 1. (a-c) Ni-catalyzed direct growth of CVD graphene on $\mathrm{SiO}_{2}$. [Reproduced with permission from ref., ${ }^{39)}$ copyright 2009, Nature Publishing Group]. (d) $\mathrm{Cu} / \mathrm{Ni}$-catalyzed direct growth of graphene on $\mathrm{SiO}_{2}$ for an FET. [Reproduced with permission from ref., ${ }^{71)}$ copyright 2009, American Chemical Society]

which promotes the formation of high-quality graphene monolayers even at low temperatures, occurs more effectively. However, this process is expensive due to the use of an expensive catalyst and high time consumption.

In 2009, the first study on the large-scale direct growth of graphene based on $\mathrm{Ni}$ catalysts on $\mathrm{SiO}_{2}$ substrates using CVD was done. ${ }^{39)}$ Thin layer of Ni was deposited on top on $\mathrm{SiO}$ to act as catalyst. After graphene growth, it can be transferred by either PDMS stamp/Ni etching or lift off on target substrate by scooping. This method can be used for graphene transfer on flexible and stretchable substrates like polydimethylsiloxane (PDMS) to be used in transparent conducting film (TCF) and field-effect transistor (FET) applications (Fig. 1(a)). ${ }^{39)}$ In this study, of graphene growth on $\mathrm{SiO}_{2}$ was not the best direction; nevertheless, this pioneering study opened up a new direction for direct graphene growth on insulating substrates (h-BN, PDMS, etc.) while avoiding complex transfer processes. The light transmittance, sheet resistance, and electron mobility of the synthesized graphene were $97 \%, 650 \Omega / \mathrm{sq}$, and $3700 \mathrm{~cm}^{2} \mathrm{~V}^{-1} \mathrm{~s}^{-1}$, respectively. ${ }^{39)}$ In addition, Levendorf et al. conducted another pioneering study using solid $\mathrm{Cu}$ and $\mathrm{Ni}$ as catalysts instead of $\mathrm{Cu}$ foil under electron-beam evaporation for depo- sition of graphene on a $\mathrm{SiO}_{2}$ substrate (Fig. 1(b)). After the CVD growth, $\mathrm{Cu} / \mathrm{Ni}$ etching was performed to obtain a graphene film on the $\mathrm{SiO}_{2}$ substrate with a mobility of $700 \mathrm{~cm}^{2} \mathrm{~V}^{-1} \mathrm{~s}^{-1}{ }^{71)}$ In another report, the direct growth of graphene based on $\mathrm{Cu}$ deposition by electron-beam evaporation on quartz, sapphire, $\mathrm{SiO}_{2}$, and fused silica substrates was performed (Fig. 2(a)). ${ }^{101)}$ Catalyst evaporation and dewetting control could lead to the direct patterned growth of graphene and other 2D materials for large-scale electronics. Teng et al. (Fig. 2(b)) presented an interesting direct CVD technique for growth of graphene with high crystallinity on $\mathrm{SiO}_{2}$, comparable to the crystallinity of graphene/Cu foil. The electron mobility $\left(100-600 \mathrm{~cm}^{2} \mathrm{~V}^{-1} \mathrm{~s}^{-1}\right)$ achieved using this technique was very low. ${ }^{78)}$ However, one problem of this method was the uniformity of the graphene layer, and it may have led to incomplete graphene coverage on the substrate.

Another effective method that has been studied is the catalytic conversion of an organic layer (a self-assembled layer or a carbon-containing organic thin-film layer) to a graphene layer at the interface between the metal catalyst film and the dielectric ionic substrate to directly grow graphene on dielectric substrates. ${ }^{72,94)}$ This method enables efficient cata- 

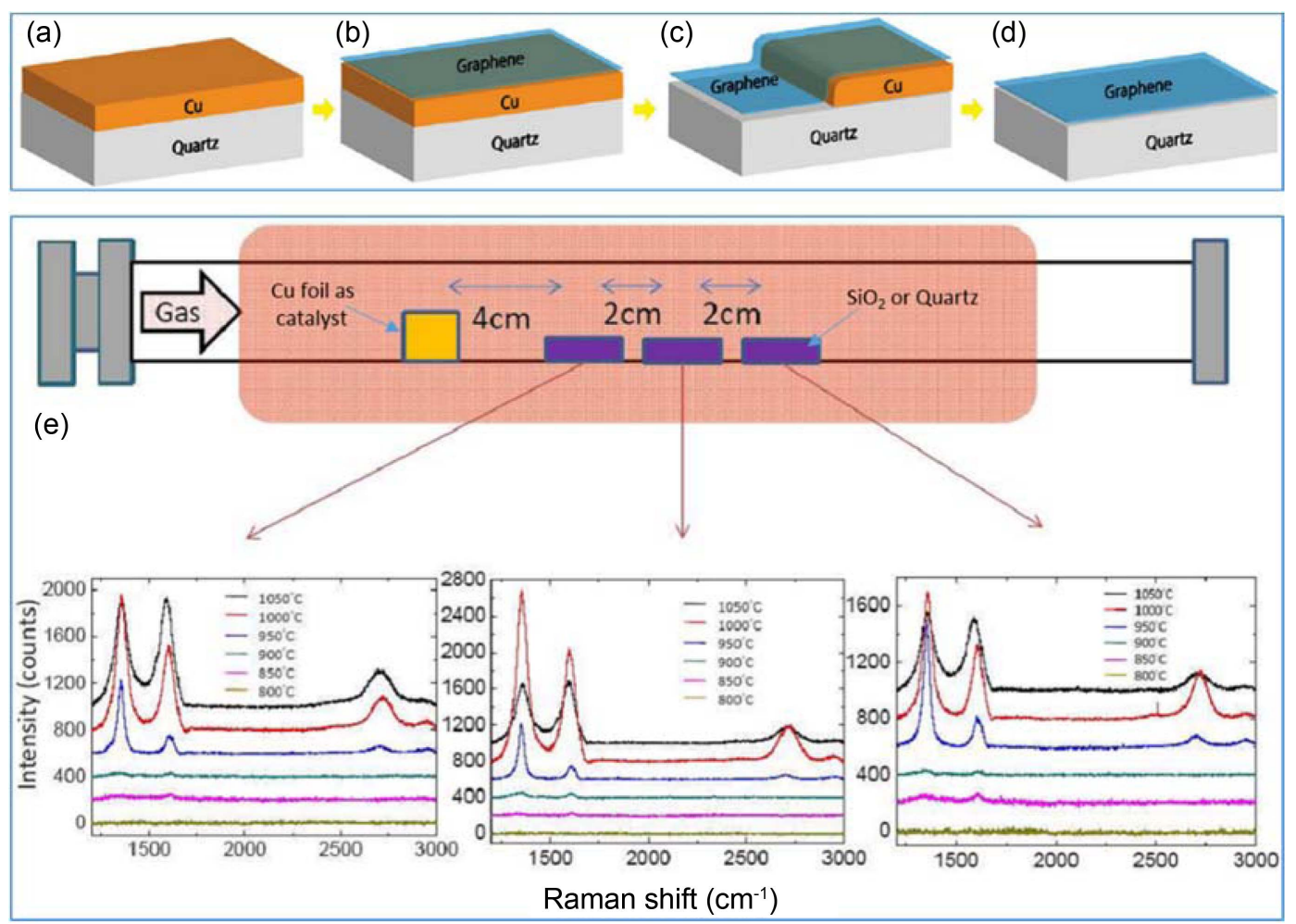

Fig. 2. (a)-(d) Cu-catalyzed direct growth of graphene on quartz substrate. [Reproduced with permission from ref., ${ }^{101)}$ copyright 2010, American Chemical Society]. (e) Cu-catalyzed direct growth of graphene films on $\mathrm{SiO}_{2}$ and quartz and their Raman data at various locations in CVD chamber. [Reproduced with permission from ref., ${ }^{78)}$ copyright 2012, American Chemical Society]

lytic conversion, allowing the direct growth of low-defectdensity graphene on dielectric substrates. However, the non-uniformity of the grown graphene layer is a drawback. Shin et al. adopted a simple transition-free synthesis of fewlayer graphene (FLG) on $\mathrm{SiO}_{2}$ substrates through the thermal decomposition of a carbon-based aliphatic self-assembled monolayer (SAM) polymer using $\mathrm{Ni}$ metal as the catalyst, as shown in Fig. $3^{70)}$ Because the amount of carbon introduced can be controlled, SAM is considered to be more effective and promising than carbon sources. This Ni/SAM/ substrate structure had an advantage in that it could robustly remove the top catalyst during the synthesis process, thus enabling the evaporated SAM chemicals to form high-quality defect-free graphene due to the high-temperature heat treatment. In 2011 Byun et al. demonstrated that catalytic metals such as $\mathrm{Ni}$ and $\mathrm{Cu}$ can be used for the cata-
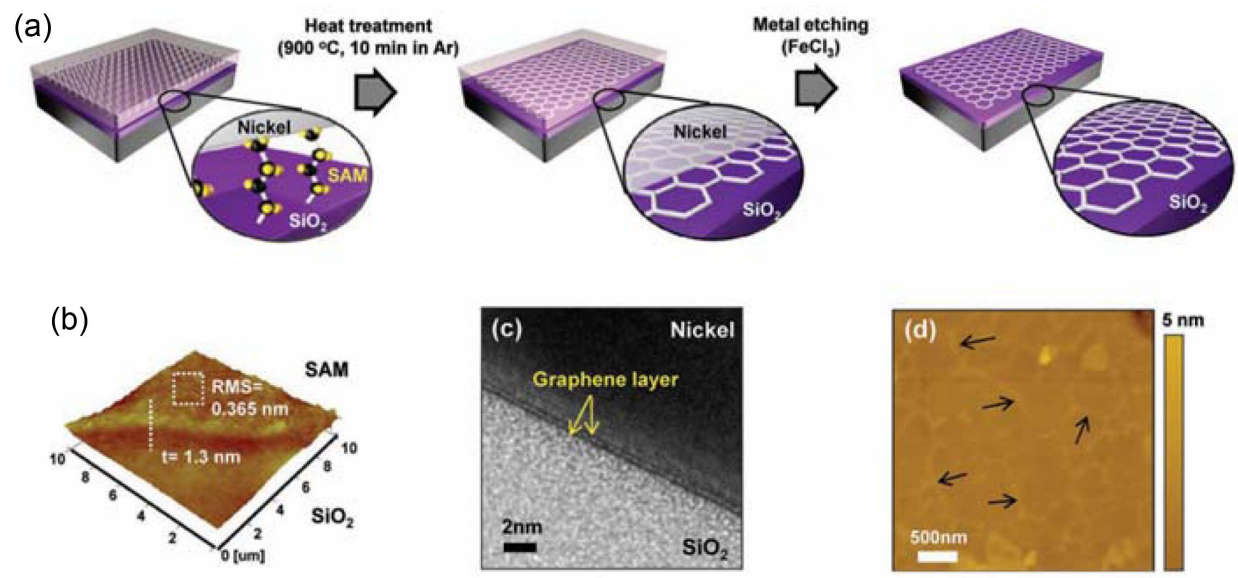

Fig. 3. (a) Ni- and SAM-catalyzed transfer-free growth of graphene on $\mathrm{SiO}_{2}$, (b) AFM image of graphene obtained from octyl-SAM on $\mathrm{SiO}_{2}$, (c) TEM image of BLG after octyl-SAM pyrolysis, and (d) AFM image of graphene/SiO 2 after Ni etching. [Reproduced with permission, Copyright 2011, WILEY-VCH Verlag GmbH \& Co. KGaA, Weinheim; Reproduced with permission from ref., ${ }^{70)}$ copyright 2012, American Chemical Society] 


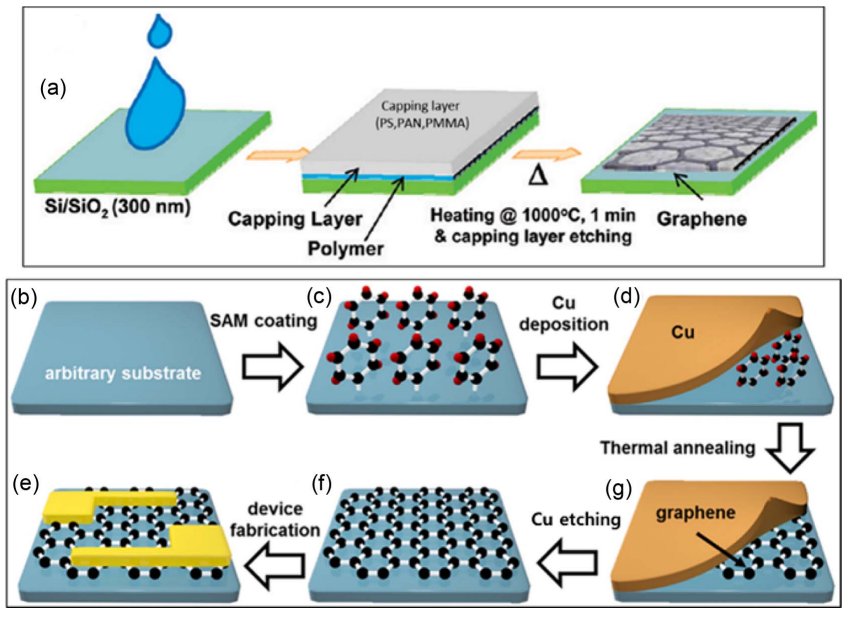

Fig. 4. (a) Transfer-free graphene growth on SAM polymers (PS, PAN, PMMA) on $\mathrm{SiO}_{2}$ substrate. [Reproduced with permission from ref., ${ }^{72}$ copyright 2011, American Chemical Society]. (b-g) Transfer-free graphene growth: (b) target substrate, (c) phenyl-SAM coating on arbitrary substrate, (d) evaporation of $\mathrm{Cu}$ thin film on SAM-coated substrate, (e) graphene growth between $\mathrm{Cu}$ layer and target substrate by thermal annealing, (f) selective $\mathrm{Cu}$ etching, and (g) graphenebased device after contact metal deposition. [Reproduced with permission from ref., ${ }^{94)}$ copyright 2016 , American Chemical Society]

lytic conversion of polymer films (polystyrene (PS), polyacrylonitrile (PAN), polymethylmethacrylate (PMMA)) (Fig. $4(\mathrm{a}))^{72)}$ These polymers are generally available at a low cost and can be used as carbon gas sources during CVD. Using these carbon sources, catalytic pyrolysis $\left(1 \mathrm{~min}\right.$ at $\left.1000^{\circ} \mathrm{C}\right)$ was performed with a $\mathrm{Ni}$ capping film (50 $\mathrm{nm}$ thick) used as the catalyst to obtain FLG. More recently, the successful synthesis of FLG exhibiting a light transmittance and sheet resistance of $84.3 \%$ and $3.5 \mathrm{k} \Omega / \mathrm{sq}$, respectively, on a desired substrate $\left(\mathrm{SiO}_{2}\right.$, quartz, GaN, etc.) was achieved using $\mathrm{Cu}$ assisted catalytic conversion of a phenyl-THF polymer (PhSi (OMe)) (Fig. 4(b)). ${ }^{94)}$ This directly grown graphene also exhibited excellent chemical sensitivity to $\mathrm{NO}_{2}$ and $\mathrm{NH}_{3}$.

Direct growth of FLG from amorphous carbon sources on sapphire substrates via $\mathrm{Ni}$ and tungsten using a thermal evaporation and annealing method was achieved by Yamada et al. (Fig. 5(a)). ${ }^{82}$ According to this strategy, deposited graphene was sandwiched between $\mathrm{W}$ and Ni layers on the top and sapphire on the bottom, as shown in the Raman and differential interference microscopy photographs. ${ }^{82)}$ In another report, graphene was grown directly on quartz and $\mathrm{SiO}_{2}$ via CVD using $\mathrm{Cu}$ as the catalyst (Fig. $5(b)) .{ }^{83)}$ This was achieved by the careful optimization of the CVD growth parameters, and the obtained graphene had a mobility of $672 \mathrm{~cm}^{2} \mathrm{~V}^{-1} \mathrm{~s}^{-1}$, which is suitable for FET applications. ${ }^{83)}$ In another pioneering study, the direct growth of high-quality bilayer graphene (BLG) on $\mathrm{SiO}_{2}$ assisted by $\mathrm{Ni}$ catalyst and carbon sources (PMMA, $\mathrm{C}_{8} \mathrm{H}_{8}, \mathrm{C}_{3} \mathrm{H}_{3} \mathrm{~N}, \mathrm{C}_{4} \mathrm{H}_{6}$ ) was described (Fig. 6) ${ }^{84}$ At a high temperature $\left(1000^{\circ} \mathrm{C}\right)$, the carbon source deposited on the Ni film decomposed and diffused into the Ni layer. When cooled to room temperature, BLG was formed between the catalyst and the substrate. Finally, after the Ni film was etched, BLG was directly obtained on $\mathrm{SiO}_{2}$ without the need for a transfer process. The growth of $\mathrm{BLG}$ on $\mathrm{SiO}_{2}$ substrates was investigated by Raman spectroscopy and transmission electron microscopy (TEM) images. ${ }^{84}$ This approach could lead to BLG-based applications in the near future.

In 2011, Liu et al. selected h-BN as the dielectric substrate for the direct synthesis of highly oriented pyrolytic graphite and mechanically peeled graphene by a two-step CVD method (Fig. 7(a)-(c)). ${ }^{86)} \mathrm{h}-\mathrm{BN}$ is an ideal dielectric substrate because it is smoother than $\mathrm{SiO}_{2}$ wafer substrates, as

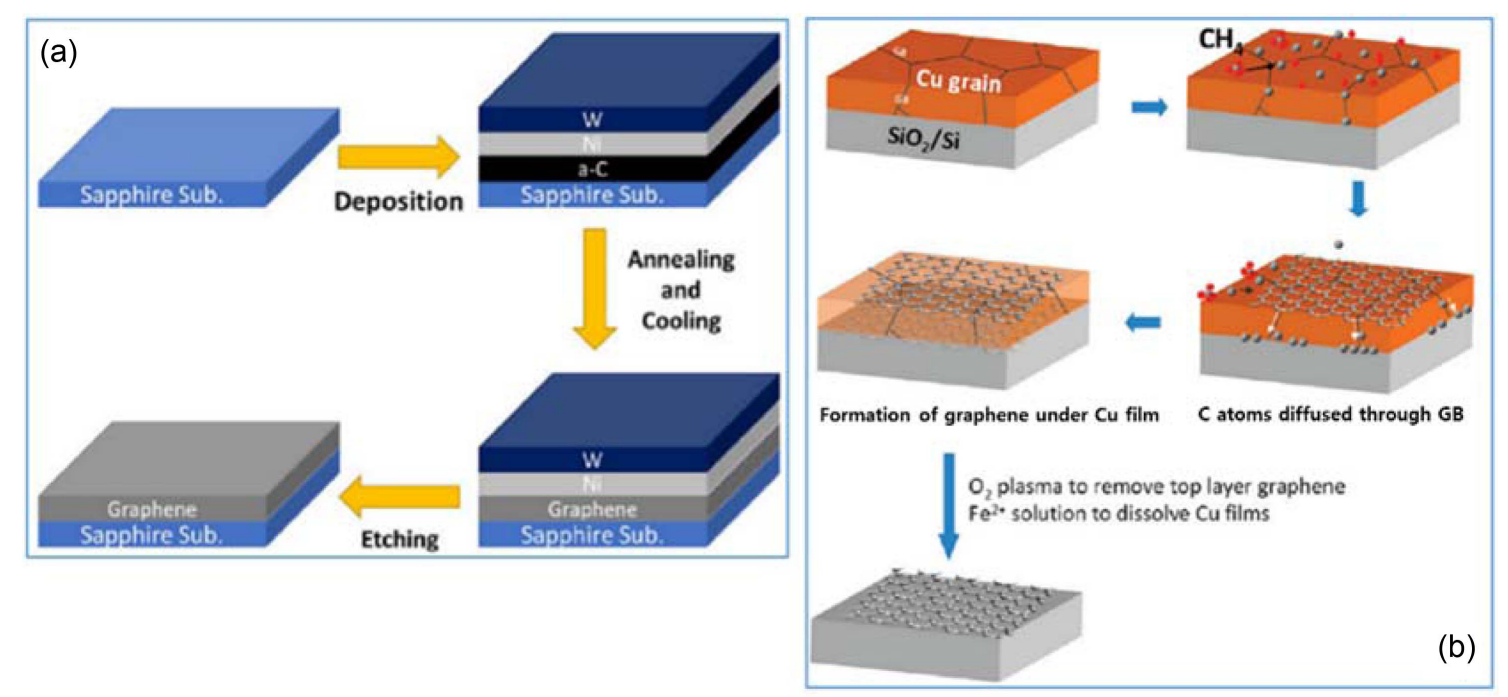

Fig. 5. (a) W- and Ni-catalyzed direct growth of graphene on sapphire substrate. [Reproduced with permission from ref., ${ }^{82)}$ copyright 2016, IOP Publishing]. (b) Cu-catalyzed direct growth of graphene on $\mathrm{SiO}_{2}$ substrate. [Reproduced with permission from ref., ${ }^{83}$ copyright 2011, American Chemical Society] 


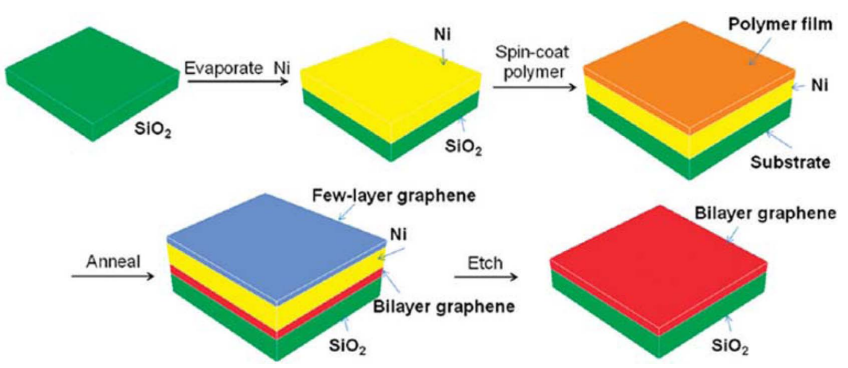

Fig. 6. Ni-catalyzed direct growth of $\mathrm{BLG}$ on $\mathrm{SiO}_{2}$ substrate from solid carbon sources by CVD. [Reproduced with permission from ref., ${ }^{84)}$ Copyright 2011, American Chemical Society]

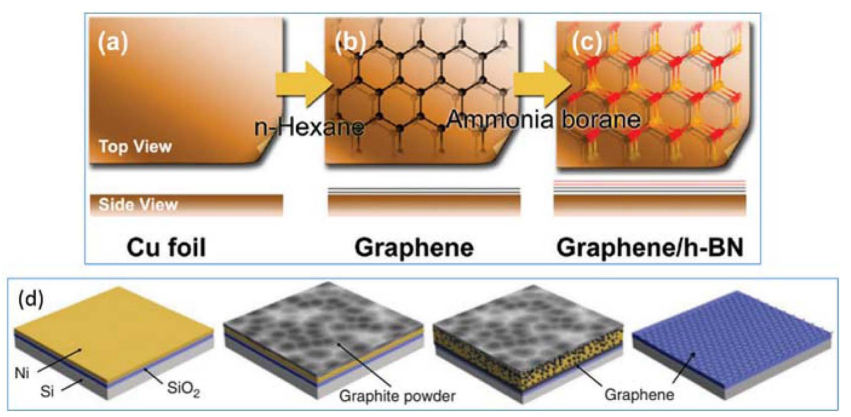

Fig. 7. (a)-(c) $\mathrm{Cu}$-, hexane-, and ammonia borane-catalyzed direct growth of CVD graphene on h-BN substrate located on $\mathrm{SiO}_{2}$. [Reproduced with permission from ref., ${ }^{86)}$ Copyright 2011, American Chemical Society]. (d) Ni-catalyzed direct growth of CVD graphene on $\mathrm{SiO}_{2}$ substrate. [Reproduced with permission from ref. ${ }^{89)}$ Copyright 2012, Nature Publishing Group]

shown by atomic force microscopy (AFM) images (scan range: $100 \mathrm{~nm}$ ) of graphene/h-BN and graphene/ $\mathrm{SiO}_{2}$ (with surface roughness values of 0.21 and $0.94 \mathrm{~nm}$, respectively). ${ }^{102)} \mathrm{CuH}_{3}$, hexane solution $\left(\mathrm{C}_{6} \mathrm{H}_{14}\right)$, and ammonia borane $\left(\mathrm{NH}_{3}-\mathrm{BH}_{3}\right)$ were also used as precursors for largescale synthesis. This approach has opened a new path for exploring the nanotechnology applications of heterostructured graphene/h-BN. Due to the high cost of growing graphene using high-temperature CVD, scientists have attempted to synthesize graphene at very low temperatures both directly and indirectly. However, this aim has faced many difficulties due to the incomplete natural growth of graphene at very low temperatures. Recently, direct growth on $\mathrm{SiO}_{2}$ near room temperature $\left(25-160^{\circ} \mathrm{C}\right)$ assisted by $\mathrm{Ni}$ catalysts has been reported ${ }^{89)}$ (Fig. 7(d)). However, the resulting graphene is of very poor quality.

In principle, it is promising to directly grow graphene on dielectric surfaces using a gaseous catalyst, since low-temperature catalyst growth of low-defect graphene is possible without further etching of the catalyst.

In 2013, Kim et al. reported SLG growth on $\mathrm{SiO}_{2}$ by copper vapor-assisted CVD (Fig. 8(a)-(d)). ${ }^{91}$ In a more recent report, $\mathrm{Li}$ et al. used $\mathrm{SiH}_{4}$ as a catalyst for direct graphene growth by CVD. ${ }^{92)}$ The $\mathrm{SiH}_{4}$ aided the direct deposition of graphene on $\mathrm{h}-\mathrm{BN}$ and $\mathrm{h}-\mathrm{BN} / \mathrm{SiO}_{2}$ insulating surfaces and
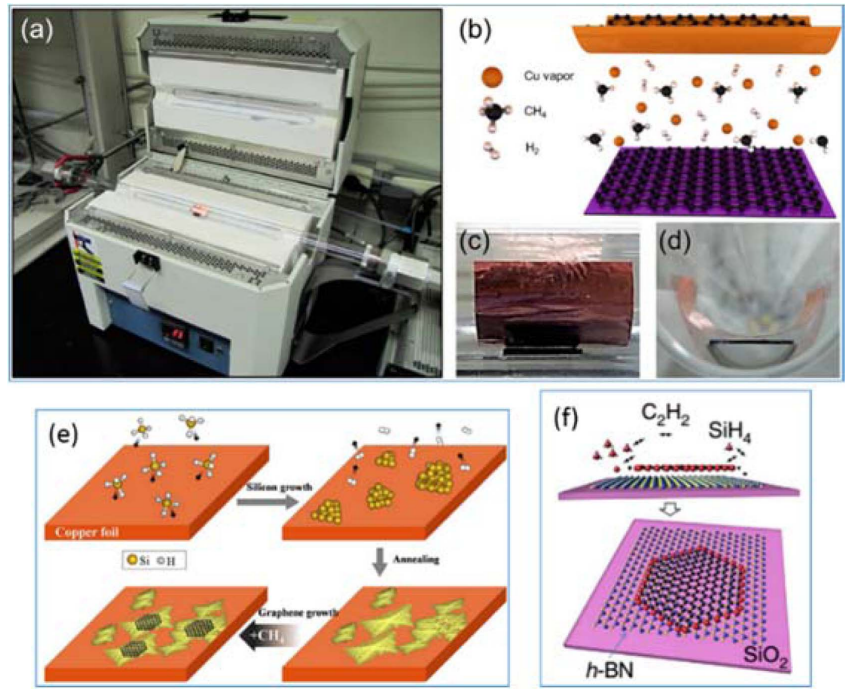

Fig. 8. (a)-(d) Cu-catalyzed direct growth of graphene on $\mathrm{SiO}_{2}$ substrate using CVD. [Reproduced with permission from ref., ${ }^{91)}$ Copyright 2013, American Chemical Society]. (e) $\mathrm{SiH}_{4}{ }^{-}$and $\mathrm{Cu}$-catalyzed direct growth of graphene on $\mathrm{SiO}_{2}$. [Reproduced with permission from ref., ${ }^{92)}$ Copyright 2016, AIP Publishing; Reproduced with permission, Copyright 2016, AIP Publishing]. (f) $\mathrm{SiH}_{4}$-catalyzed growth of SLG on h-BN substrate located above $\mathrm{SiO}_{2}$ wafer. [Reproduced with permission from ref., ${ }^{93)}$ Copyright 2015, Nature Publishing Group]

the assembly of $\mathrm{Cu}$ foil and $\mathrm{SiH}_{4}$ catalyst inside the CVD chamber (Fig. 8(e)). The best results were obtained by Tang et al. at a growth rate $\sim 1 \mu \mathrm{m} / \mathrm{min}$ with a graphene domain size of $\sim 20 \mu \mathrm{m}$ and mobility of $\sim 20,000 \mathrm{~cm}^{2} \mathrm{~V}^{-1} \mathrm{~s}^{-1}$ (Fig. $8(f)) .^{93)}$

Direct growth of graphene on flexible organic substrates is highly desirable for applications related to flexible and stretchable electronics such as e-skins, biosensors, and health monitors for humans. ${ }^{103,104)}$ However, the limited thermal stability of the organic substrates, which are easily melted, deformed, or damaged at high temperatures $\left(>300^{\circ} \mathrm{C}\right)$, is a serious limitation. So far, the quality of graphene grown at low temperatures $\left(<400^{\circ} \mathrm{C}\right)$ is much poorer than that at high temperatures $\left(\sim 1000^{\circ} \mathrm{C}\right)$. Due to these constraints, research on graphene growth on flexible substrates has only begun recently. ${ }^{80,81)}$ To reduce the process temperature, most of the studied growth methods involve the catalytic conversion of organic precursors to graphitic layers on flexible organic substrates with the help of catalytic metal layers. In 2012, Kim et al. revealed a lowtemperature $\left(300^{\circ} \mathrm{C}\right)$ growth method using inductively coupled PECVD and a direct transfer of graphene-graphitic carbon (G-GC) films onto a flexible polyimide (PI) substrate with no additional chemicals (Fig. 9(a)-(f)). ${ }^{80)}$ The optical and electrical characteristics of G-GC were affected by varying growth temperature, plasma power, and growth time. Due to the strong van der Waals forces and $\pi-\pi$ interactions at the interfaces between the G-GC films and the PI sub- 
(a)

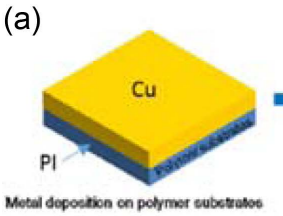

(b)

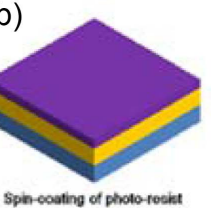

(f)

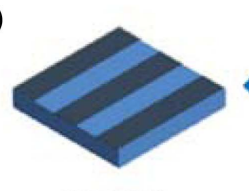

(e)

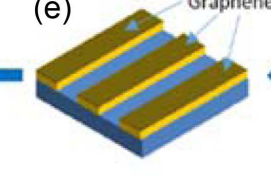

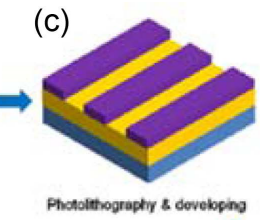

(d)

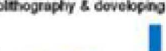

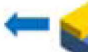

Metal etching 8 L in on

(g)
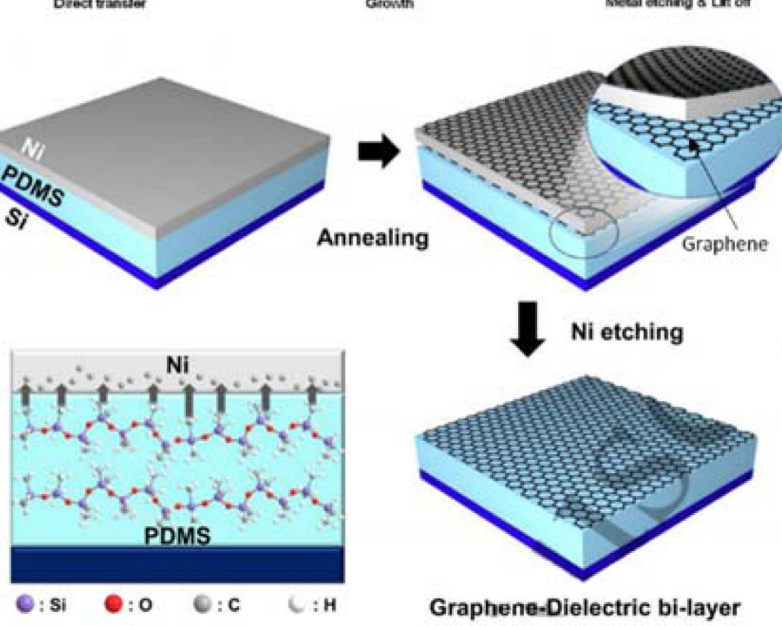

Fig. 9. Direct growth of graphene on flexible substrates: (a)(f) PI. [Reproduced with permission from ref., ${ }^{80)}$ copyright 2012, IOP Publishing]. (g) Direct growth of BLG on PDMS substrate using Ni catalyst. [Reproduced with permission from ref., ${ }^{81}$ copyright 2017 , IOP Publishing]

strate, the patterned G-GC films were not facially detached or damaged on the substrate after wet etching and transferring. More recently, in 2016, Seo et al. revealed a simple, scalable, inexpensive, and patternable process to synthesize graphene-dielectric bilayer (GDB) films from solution-processed PDMS under a Ni capping layer (Fig. 9(g)). ${ }^{81)}$ Here, $\mathrm{Ni}$ films were deposited as the catalyst and the encapsulation layer on the PDMS layer had a thickness of a few micrometers. This layer enabled the direct growth of GDB between the substrate and the Ni layer. PDMS $(4 \mu \mathrm{m}) / \mathrm{Ni}$ $(400 \mathrm{~nm})$ films on the substrate were thermally annealed using a vacuum furnace to form a PDMS/multilayer graphene (MLG)/Ni/MLG structure. At the interface of the PDMS layer and the Ni film, the carbon atoms on the PDMS surface diffused into the Ni layer at high temperatures, and the carbon atoms were released to form MLG on both sides of the Ni layer during cooling. This method was used to fabricate GDB simultaneously and directly on the substrate by the thermal conversion of PDMS without using additional graphene transfer and patterning processes or without forming an expensive dielectric layer, thus making the device fabrication process much easier.

\subsection{Non-catalytic growth}

Direct growth of graphene on arbitrary substrates without the need of an additional catalyst is advantageous due to the fewer processing steps required and lower processing cost. However, this approach is limited by low activation energy; as a result of this low energy, chemical reactions related to graphene growth cannot successfully obtain uniform high-quality graphene for commercialization compared to existing synthesis processes. In 2011, Zhang et al. reported the use of a remote PECVD system at low temperature $\left(500^{\circ} \mathrm{C}\right)$ for the direct growth of graphene on various inorganic dielectric substrates (sapphire, quartz, mica, Si, SiC, glass) (Fig. 10(a)). ${ }^{68)}$ This method was cost-effective, simple, scalable (up to 4 -inch wafers), and compatible with devices such as nanoresistors, gas sensors, and TCFs. In another report, Song et al. demonstrated catalyst-free direct growth of large-scale SLG on sapphire without graphene transfer using high-temperature $\left(950^{\circ} \mathrm{C}\right) \mathrm{CVD}$ (Fig. 10(b)) ${ }^{76)}$ In 2011, Son et al. reported catalyst-free direct growth of graphene in a horse-pad shape with a diameter of up to $\sim 110 \mathrm{~nm}$ on a mechanically exfoliated h-BN surface placed on a $\mathrm{SiO}_{2}$ substrate through ambient-pressure CVD (APCVD) (Fig. 11(a)-(h)). ${ }^{105)}$ Moreover, this study indicated that graphene growth involved nucleate particle formation, and BLG was formed as an instantaneous state during SLG
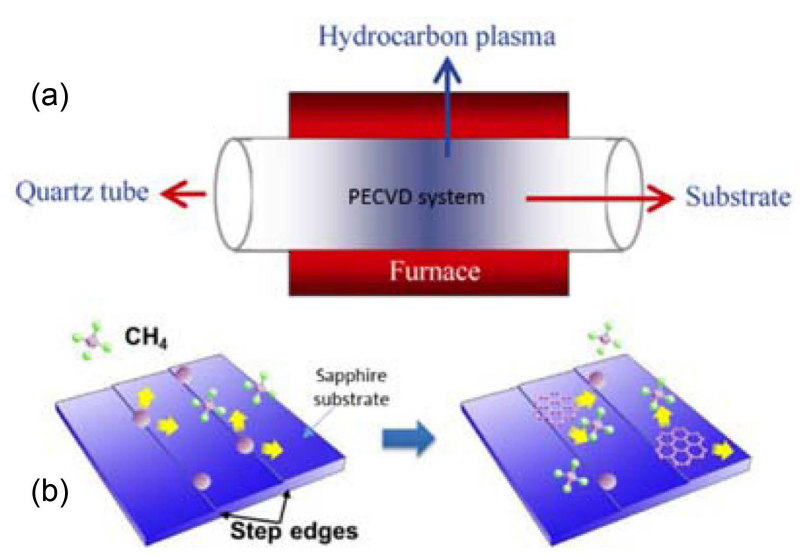

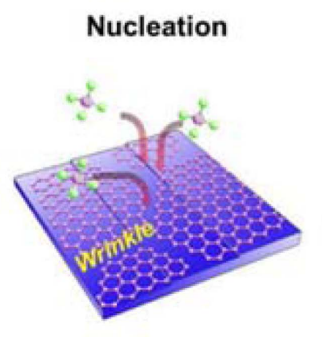

Graphene film growth

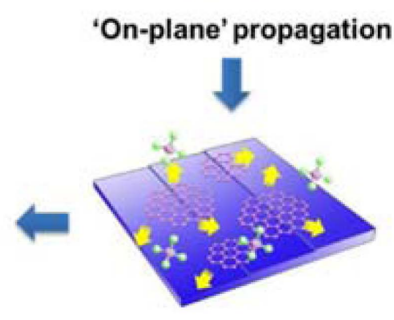

Graphene dot growth
Fig. 10. (a) PECVD system for graphene film growth. [Reproduced with permission from ref. ${ }^{68)}$ Copyright 2011, Springer and Tsinghua University Press]. (b) Catalyst-free direct growth of graphene on sapphire via CVD. [Reproduced with permission from ref., ${ }^{76)}$ Copyright 2012, The Royal Society of Chemistry] 


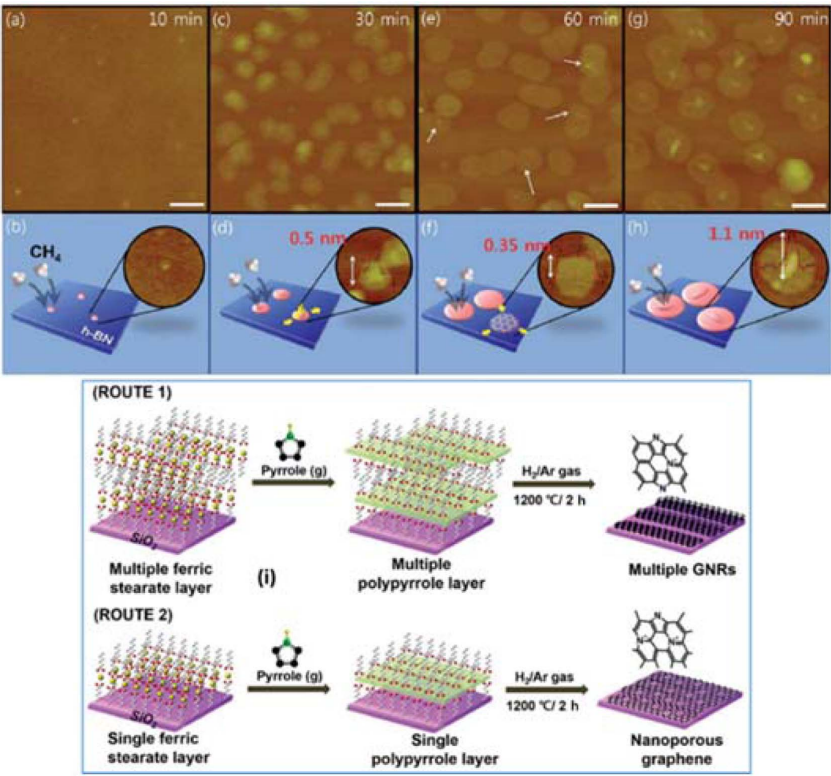

Fig. 11. (a)-(h) Catalyst-free direct growth of graphene pad on $\mathrm{h}-\mathrm{BN} / \mathrm{SiO}_{2}$ substrate. [Reproduced with permission from ref., ${ }^{105)}$ Copyright 2011, The Royal Society of Chemistry]. (i) Catalyst-free bottom-up graphene growth on $\mathrm{SiO}_{2}$ substrate. [Reproduced with permission from ref., ${ }^{85)}$ Copyright 2016, The Royal Society of Chemistry]

growth on h-BN via a peripheral process. ${ }^{105)}$ Most recently, Min et al. reported a new technique for catalyst-free bottomup growth of graphene nanoribbon (GNRs) and nanoporous sheets based on the pyrolysis of SAMs of ferric stearate molecules directly onto $\mathrm{SiO}_{2}$ substrates. ${ }^{85)}$ The GNRs containing pyrrolic $\mathrm{N}$-enriched edges obtained by this method exhibited p-type semiconductor properties, whereas the nanoporous graphene sheets containing inhomogeneous pores and graphitic N-enriched basal planes exhibited n-type semiconductor properties (Fig. 11(i)). ${ }^{85)}$ It was expected that this direct graphene growth technique could be applied to various graphene-based electronic applications without the requirement for graphene transfer. However, the drawback of this method was the high-density defects of the GNRs, which were confirmed by the high $\mathrm{D}$ peak in their Raman spectra. ${ }^{85)}$

In 2016, Song et al. reported the direct growth of conformal graphene films on 3D quartz surfaces, which could be applied to pressure sensors. ${ }^{55}$ ) This $3 \mathrm{D}$ graphene layer grown on the quartz surface had excellent electrical and optical properties, with a sheet resistance of $\sim 2000 \Omega / \mathrm{sq}$ and light transmittance of $\sim 80 \%$ at $550 \mathrm{~nm}$, which could be attributed to the presence of a flat graphene film on the PDMS substrate; the obtained film could be used as a pressure-sensitive sensor. Trung et al. ${ }^{87)} \mathrm{Xu}$ et al. ${ }^{88)}$ and $\mathrm{Bi}$ et $a l .{ }^{90)}$ showed the direct growth of graphene on $\mathrm{Si}, \mathrm{SiO}_{2}$, quartz, AlN, and h-BN substrates without a catalyst support. Xu et al. used CVD with two temperature zones for the direct growth of uniform and continuous graphene layers,

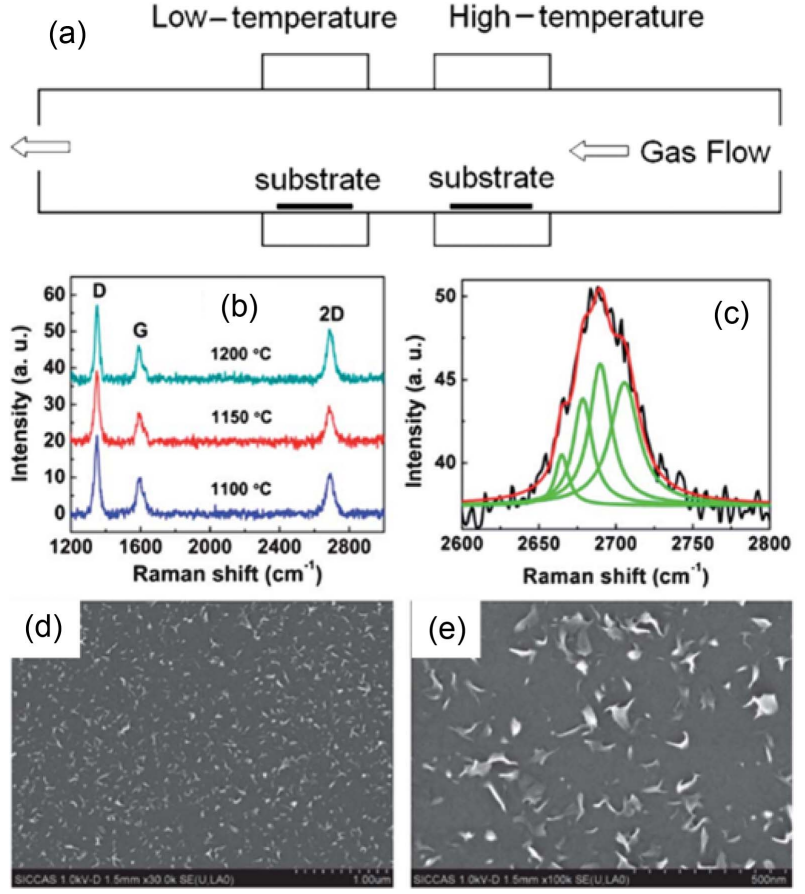

Fig. 12. (a) Direct growth of graphene on $\mathrm{SiO}_{2}$ by two-temperature-zone CVD. [Reproduced with permission from ref., ${ }^{88}$ Copyright 2013, The Royal Society of Chemistry]. (b)-(e) Direct growth of $\mathrm{FLG}$ on $\mathrm{SiO}_{2}$ : (b) Raman spectra of directly grown graphene/ $\mathrm{SiO}_{2}$ at 1100,1150 , and $1200^{\circ} \mathrm{C}$, (c) Raman $2 \mathrm{D}$ peaks, and d, (e) SEM images. [Reproduced with permission from ref., ${ }^{90)}$ Copyright 2012, The Royal Society of Chemistry]

as shown in Fig. 12(a). ${ }^{88)}$ High-temperature $\mathrm{CH}_{4}$ dissociation provided the active carbon species required for low-temperature graphene growth. As a result, the graphene grown directly on the transparent quartz substrate showed a reasonably good light transmittance and sheet resistance of $97 \%$ and $650 \Omega /$ sq, respectively. ${ }^{88)}$ These values reported by $\mathrm{Xu}$ et al. were almost similar to those reported by Bi et al. (94\% and $425 \Omega /$ sq, respectively, using PECVD) (Fig. 12(b)(e)). ${ }^{90)}$ These CVD and PECVD techniques could synthesize transfer-free graphene for future high-performance electronic devices. In another report, using remote electron cyclotron resonance plasma-assisted CVD $\left(650^{\circ} \mathrm{C}\right)$, Munoz et al. directly grew graphene on insulating substrates (quartz, $\mathrm{SiO}_{2}$ ) at low temperature. ${ }^{97)}$ Through the sophisticated control of the growth parameters (e.g., nucleation density, edge growth, thickness), Munoz et al. obtained highly crystalline graphene monolayers exhibiting a light transmittance of $\sim 92 \%$ and sheet resistance of $\sim 900 \Omega /$ sq. $^{97)}$ In early 2017 , Pang et al. reported a new method based on direct self-terminating confinement on two sandwiched $\mathrm{Si} / \mathrm{SiO}_{\mathrm{x}}$ substrates for large-scale growth of uniform graphene monolayers (Fig. 13) ${ }^{98)}$ This result is a significant advancement for transfer-free graphene growth, considering that the transfer-free synthesis of "extremely uniform" graphene as a monolayer or bilayer is a significant challenge. 


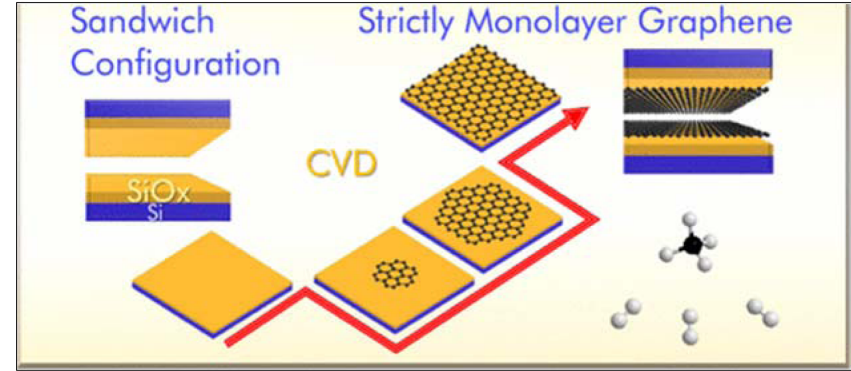

Fig. 13. Direct growth of CVD-based large-area uniform SLG on $\mathrm{SiO}_{2}$. [Reproduced with permission from ref., ${ }^{98)}$ Copyright 2017, American Chemical Society].
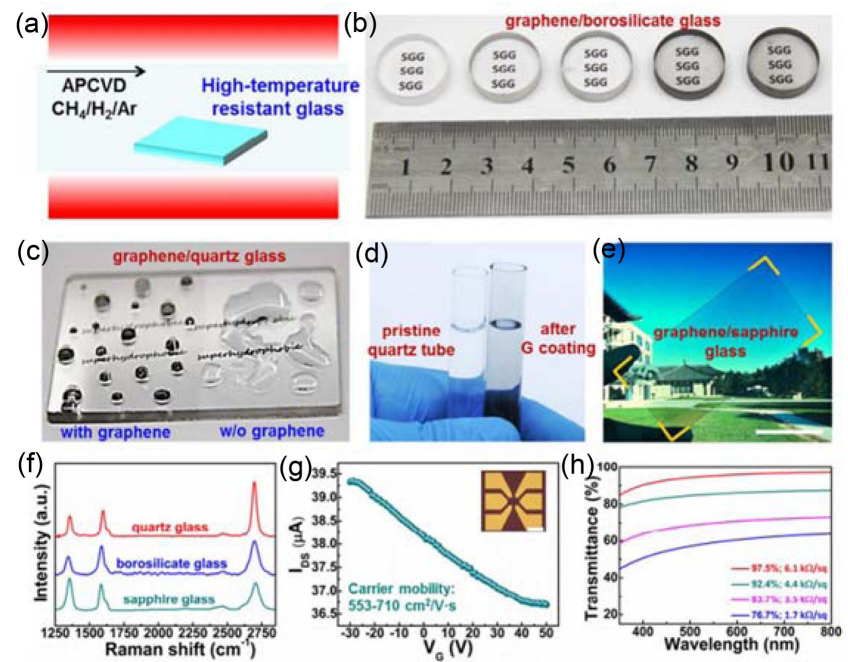

Fig. 14. Catalyst-free APCVD growth of uniform graphene on various solid glass substrates (quartz, borosilicate, sapphire). [Reproduced with permission from ref., ${ }^{99)}$ Copyright 2015, American Chemical Society].

In 2015, Sun et al. reported the catalyst-free direct growth of uniform graphene on various solid glass substrates (quartz, borosilicate, sapphire) using cost-effective APCVD and its potential for daily-life applications such as heatresistant devices, photocatalytic plates, and energy-saving smart windows, as shown in Fig. $14{ }^{99)}$ This is one of the pioneering studies for the direct well-controlled large-scale growth of high-quality graphene on insulating solid glass. Sun et al. discovered that the light transmittance and sheet resistance values of the synthesized graphene could be facially compatible together with the experimentally tunable layer thickness of graphene. ${ }^{99)}$ In 2010, Rummeli et al. investigated a new facile strategy for the direct growth of graphene on a $\mathrm{MgO}$ dielectric substrate using hexane $\left(\mathrm{C}_{6} \mathrm{H}_{14}\right)$ as the catalyst via very-low-temperature $\left(325^{\circ} \mathrm{C}\right)$ CVD. ${ }^{67)}$ These graphene films were well formed and confirmed $\mathrm{MgO}$ to be a suitable dielectric substrate for direct graphene synthesis via $\mathrm{CVD}$ at $325^{\circ} \mathrm{C}$ without the requirement for a transfer process. This approach has considerable potential for large-scale fabrication of future Si-assisted devices.

\section{Low-temperature Growth}

So far, most graphene growth processes have used methane as the precursor to produce high-quality graphene monolayers at temperatures of $\sim 1000^{\circ} \mathrm{C}$. To overcome the difficulties encountered during high-temperature growth processes using methane, the use of other solid or liquid hydrocarbon precursors has recently received considerable attention. As a result, the growth temperature of graphene has greatly decreased to as low as $300^{\circ} \mathrm{C}$ by adopting alternative carbon sources such as hexane, ethanol, ethylene, pentane, alcohol, and benzene for thermal CVD and PECVD. The obtained graphene structures have been analyzed using advanced techniques and have been adopted for the fabrication of multifunctional devices.

\subsection{Adoption of liquid precursors}

Graphene monolayers were obtained using thermal CVD at a growth temperature of around $1050^{\circ} \mathrm{C}$ and ultra-low pressure. ${ }^{61,106)}$ Further, graphene monolayers with a similar quality were synthesized by Zhang et al. using toluene as the precursor via low-pressure CVD at a low growth temperature of $600^{\circ} \mathrm{C} .^{107)}$ In this study, copper foil, which was used as the substrate, was electrochemically polished before deposition and ultrahigh-purity hydrogen and toluene were used at flow rates of $3.3 \mathrm{sccm}$ and $2.7 \mathrm{mLh}^{-1}$ respectively. The partial pressure was maintained at $300 \mathrm{~m}$ Torr during the reaction. The $\mathrm{Cu}$ thin film was annealed at $980^{\circ} \mathrm{C}$ for 15 min and then grown in two steps. It was grown at the desired temperature $\left(300-600^{\circ} \mathrm{C}\right)$ for $60 \mathrm{~min}$ initially and then the growth was continued at a 10-fold higher pressure maintained by closing the throttle valve for $5 \mathrm{~min}$. A continuous graphene film grown on $\mathrm{Cu}$ electropolished at $600^{\circ} \mathrm{C}$ in two stages is shown in Fig. 15; as is clearly visible in the optical image, the graphene film is transferred to a $\mathrm{Si} / \mathrm{SiO}_{2}$ substrate. According to Raman analysis, the $2 \mathrm{D} / \mathrm{G}$ peak ratio was about 2.18 and the $2 \mathrm{D}$ full maximum at half width (FWHM) value was about $36 \mathrm{~cm}^{-1}$. It indicated that the asgrown graphene was composed of a fault with considerably low defects. Confocal Raman spectroscopy mapping over a $30 \times 30 \mu^{2}$ region confirmed that the as-grown structure was a single layer. FETs with a channel width of $300 \mathrm{~mm}$ exhibited depletion-type characteristics with p-type conductivity. The electron and hole mobilities of these graphene samples were measured to be 190 and $811 \mathrm{~cm}^{2} \mathrm{~V}^{-1} \mathrm{~s}^{-1}$, respectively. This structure exhibited a light transmittance of $97.33 \%$ at a wavelength of $550 \mathrm{~nm}$ and a sheet resistance of $8.02 \times 10^{3} \Omega /$ sq.

Jang et al. ${ }^{108)}$ demonstrated the growth of a continuous SLG structure on a copper foil substrate via oxide-free CVD using benzene as the precursor. One of the biggest obstacles to the growth of high-quality graphene without the use of amorphous or oxidized carbon layers is the presence of residual oxidizing species in the CVD growth chamber. To overcome this problem, growth was performed in an oxygenfree APCVD system. In a typical process, high-quality con- 

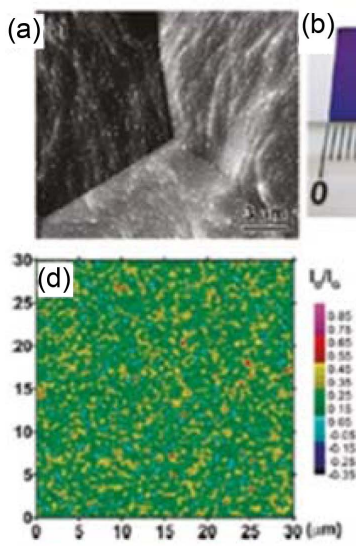
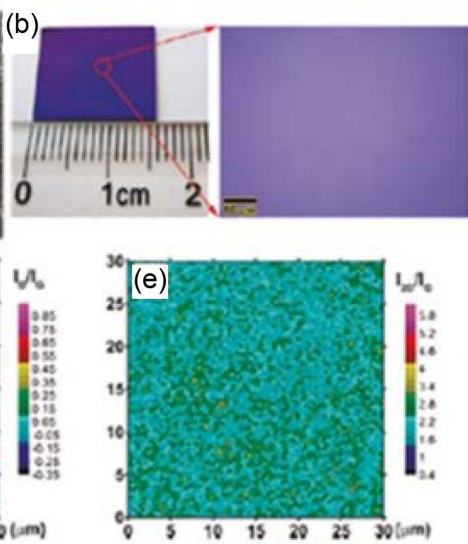
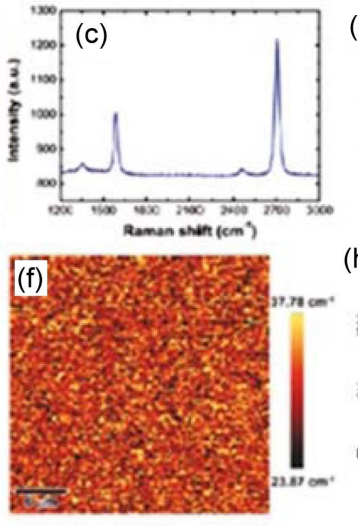
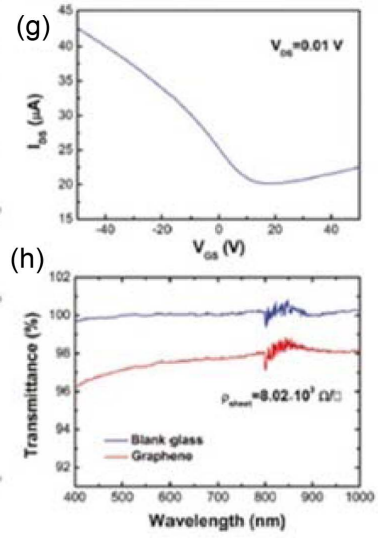

Fig. 15. Toluene-derived continuous graphene films grown at $600^{\circ} \mathrm{C}$. (a) SEM image of graphene grown on electropolished $\mathrm{Cu}$ surface, (b) optical image of graphene transferred onto $\mathrm{Si} / \mathrm{SiO}_{2}$ substrate, (c) typical Raman spectrum of graphene, (d), (e) Raman plots of intensity ratio of D band to G band and 2D band to G band, respectively, (f) Raman map of FWHM of $2 \mathrm{D}$ band (measured over $30 \times 30 \mu^{2}$ area), (g) $\mathrm{I}_{\mathrm{DS}} \mathrm{vs}$. $\mathrm{V}_{\mathrm{GS}}$ curve for a constant $\mathrm{V}_{\mathrm{DS}}$ of $0.01 \mathrm{~V}$, and (h) transmittance of graphene film from 350 to $1000 \mathrm{~nm}$. [Reproduced with permission from ref., ${ }^{107)}$ copyright 2012, American Chemical Society]

tinuous graphene sheets were synthesized on a copper foil at $300^{\circ} \mathrm{C}$. Here, the aromatic benzene molecules entering the reaction chamber were adsorbed on the surface of the copper foil, causing a catalytic dehydrogenation reaction that formed highly reactive six-ring species and graphene nuclei, as depicted in Fig. 16. Electron microscopy and optical microscopy images of the as-grown graphene showed that the growth of the graphene layer on the copper foil was continuous and uniform. Raman spectral analysis of this structure showed the presence of SLG with few defects.
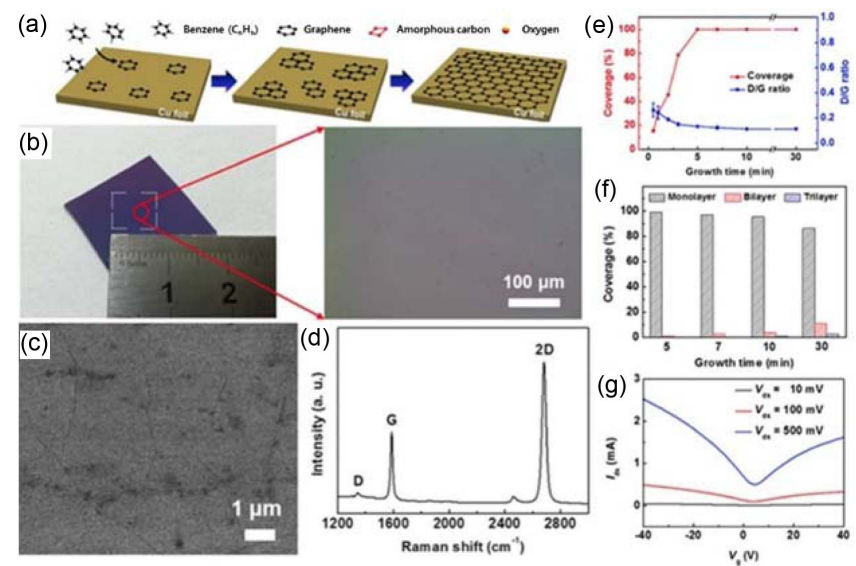

Fig. 16. (a) Mechanisms of graphene growth from benzene on $\mathrm{Cu}$ surface, (b) photograph and OM image, (c) SEM image, and d) Raman spectrum of continuous $\mathrm{SLG}$ film grown at $300^{\circ} \mathrm{C}$ for $5 \mathrm{~min}$. (e) Surface coverage and intensity ratio of $D$ band to $G$ band (D/G ratio) in Raman spectra of graphene films grown at $300^{\circ} \mathrm{C}$ for different times (30 s to $30 \mathrm{~min}$ ), (f) surface coverage distribution of graphene films grown at $300^{\circ} \mathrm{C}$ for different times $(5-30 \mathrm{~min})$, and (g) $I_{d s}-V_{g}$ characteristics of graphene FET recorded at various $\mathrm{V}_{\mathrm{ds}}$ values at room temperature in vacuum. [Reproduced with permission from ref., ${ }^{108)}$ copyright 2015, Nature Publication group]
Studies on graphene growth time showed that graphene quality and surface coverage increased with increasing growth time. The graphene structure grown for 5 min exhibited $100 \%$ surface coverage and a uniform $\mathrm{I}_{2 \mathrm{D}} / \mathrm{I}_{\mathrm{G}}$ value of $\sim 2$, but BLG and MLG flakes were formed in the monolayer over longer durations. FETs fabricated on $\mathrm{Si} / \mathrm{SiO}_{2}$ substrates with $\mathrm{Ti} / \mathrm{Au}$ contacts exhibited typical transistor characteristics. This measurement showed that the graphene structure grown at $300^{\circ} \mathrm{C}$ exhibited low sheet resistance (1 $\mathrm{k} \Omega / \mathrm{cm}$ ) and high electron and hole mobilities (1900 and $2500 \mathrm{~cm}^{2} \mathrm{~V}^{-1} \mathrm{~s}^{-1}$, respectively).

\subsection{Adoption of solid precursors}

A graphene monolayer structure was developed on a copper foil using a solid carbon source at a pressure of $8-15$ Torr and a flow rate of $50 \mathrm{sccm}$ for $45 \mathrm{~min} .{ }^{66)}$ When the precursor was changed to liquid benzene, the growth temperature significantly decreased to $300^{\circ} \mathrm{C}$, while high-quality graphene structures were produced at $800^{\circ} \mathrm{C}$ using solid PMMA (or PS). As shown in Fig. 17, a typical feedstock gas was supplied by placing a solid precursor (PMMA or PS) individually on the gas inlet side of the growth tube. Highquality SLG was synthesized at 1000 and $800^{\circ} \mathrm{C}$ using PMMA as the raw material. When the growth temperature was lower than $800^{\circ} \mathrm{C}$, a disordered hydrocarbon structure was observed together with graphene.

A mild radical-induced growth of graphene structures was achieved by Jiang et $a l .^{109)}$ at lower growth temperatures $\left(220-250^{\circ} \mathrm{C}\right)$ using a two-zone furnace. The growth of graphene over $\mathrm{Cu}(111)$ surfaces was performed by evaporating hexabromobenzene (HBB) under ultra-high vacuum conditions using a Knudsen cell. Here, HBB molecules, which were deposited at room temperature, underwent debromination through Ullmann reaction catalyzed by the copper surface, and bromine adatoms aggregated in hexagonal closely packed islands (Fig. 18). As a result, the decom- 
(a)
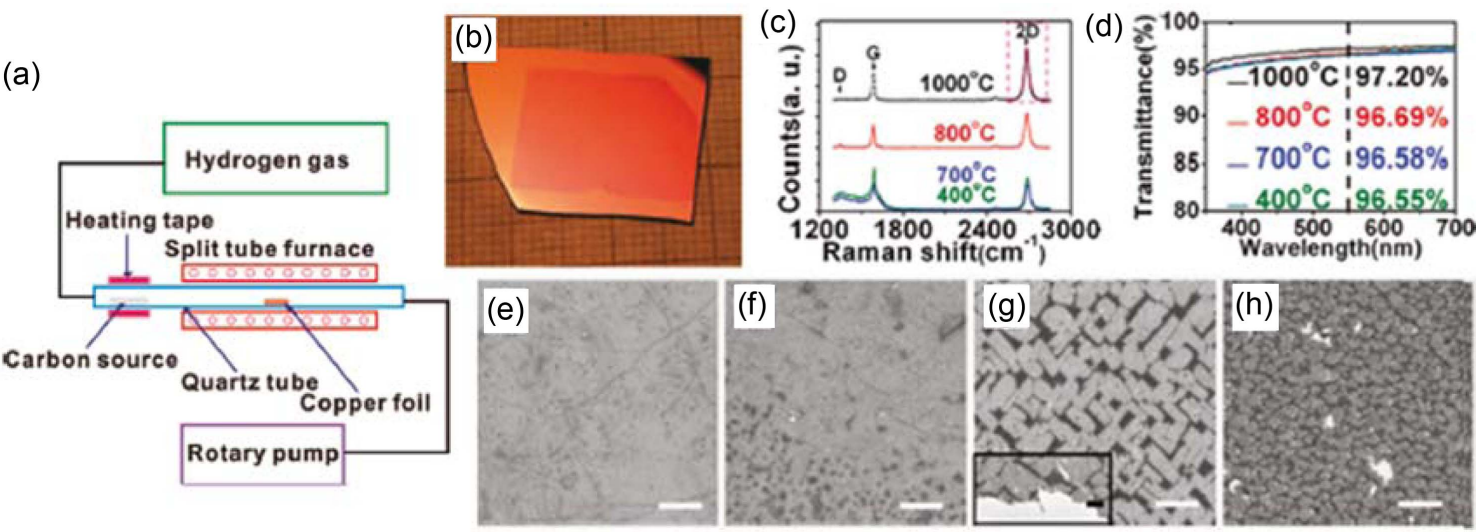

Fig. 17. (a) CVD growth using solid carbon sources on $\mathrm{Cu}$ foils. PMMA-derived graphene grown at different temperatures, (b) graphene grown at $1000^{\circ} \mathrm{C}$, (c) Raman spectra, (d) optical transmittance spectra (light transmittance at $550 \mathrm{~nm}$ for different growth temperatures are presented in the same panel), and $(\mathrm{e} \sim \mathrm{h})$ SEM images of graphene at 1000, 800, 700, $400^{\circ} \mathrm{C}$ (the black line of figure $\mathrm{g}$ is an an SEM image showing the sharp edge of graphene on $\mathrm{Si} / \mathrm{SiO}_{2}$ substrate). [Reproduced with permission from ref., ${ }^{66)}$ copyright 2011, American Chemical Society]

posed carbon atoms aggregated into disordered clusters. However, high-quality graphene structures were realized on the $\mathrm{Cu}(111)$ surface by depositing $\mathrm{HBB}$ at $570^{\circ} \mathrm{C}$. Electrical characteristics of the as-grown graphene structures evaluated using FETs fabricated on $\mathrm{Si} / \mathrm{SiO}_{2}$ substrates exhibited mobilities in the range of $1000-4200 \mathrm{~cm}^{2} \mathrm{~V}^{-1} \mathrm{~s}^{-1}$. The overall results clearly indicated the high quality of the graphene structures.
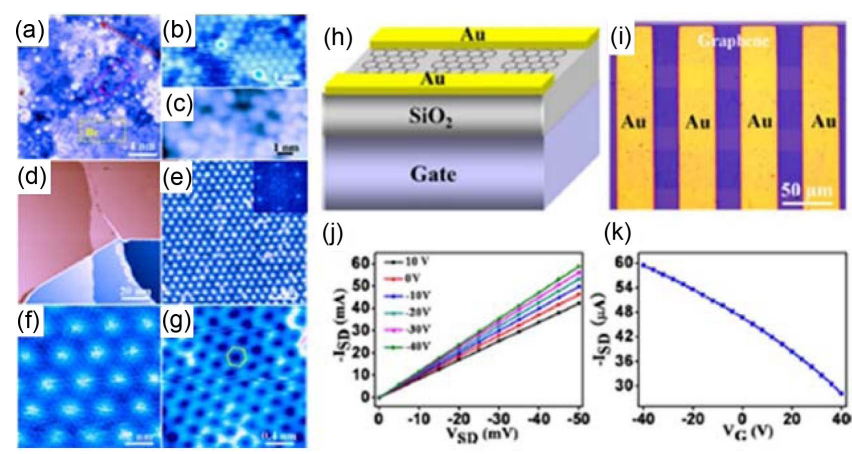

Fig. 18. STM topographies of HBB radical-coupling reactions on $\mathrm{Cu}(111)$ : (a) initial formation of HBB clus ters and bromine islands at room temperature, (b) magnified image of bromine-adatom islands with hexagonal packing, (c) magnified image of disordered aggregated carbon clusters, (d)-(f) radical coupling-assisted formation of SLG by decomposing $\mathrm{HBB}$ on annealed $\mathrm{Cu}(111)$ at $570^{\circ} \mathrm{C}$, (d) large-area scan of SLG on $\mathrm{Cu}(111)$, (e) STM image of graphene islands on $\mathrm{Cu}(111)$ (inset: Fourier transforms of hexagonal superstructure), (f) atomic-resolution STM image showing periodic spacing of $2.3 \mathrm{~nm}$ in the Moiré pattern, (g) atomic-resolution STM image of the bright spot in (f) with honeycomb structure, (h) schematic of an FET with $\mathrm{Au}$ as source and drain electrodes, (i) microscopic image of transistor arrays on graphene film, (j) graphene transistor current output at different voltages, and (k) typical output and transfer characteristics. [Reproduced with permission from ref., ${ }^{109)}$ copyright 2013, American Chemical Society]
High-quality SLG structures were obtained using carboncontaining CVD (CE-CVD) processes at very low temperatures of $500^{\circ} \mathrm{C} .{ }^{110)}$ Low-temperature CE-CVD was used for SLG growth (Fig. 19). The graphene structure grown on the entire surface of the $\mathrm{Cu}$ foil showed a very strong $\mathrm{D}$ peak, but the graphene structure on the backside of the foil had a FWHM of $\sim 35 \mathrm{~cm}^{-1}$, indicating a very strong $2 \mathrm{D}$ peak and $\mathrm{I}_{2 \mathrm{D}} / \mathrm{I}_{\mathrm{G}}$ ratio of approximately 2 . This result highlighted that the graphene structure grown on the backside of the $\mathrm{Cu}$ foil was composed of high-quality SLG. While lowering the growth temperature from $1000^{\circ} \mathrm{C}$ to $500^{\circ} \mathrm{C}$, the reduction in the $\mathrm{I}_{2 \mathrm{D}} / \mathrm{I}_{\mathrm{G}}$ ratio was negligible, indicating that the SLG quality at $500^{\circ} \mathrm{C}$ was similar to that achieved at $1000^{\circ} \mathrm{C}$. This result emphasized the importance of graphite holders for copper catalyst behavior, which greatly improved the growth of SLG.

The temperatures required for graphene growth are significantly lowered by the use of polycyclic aromatic hydrocarbons (PAHs) because the activation energy between the metal substrate and PAHs is reduced, which is important for the dehydrogenation of precursors and subsequent nucleation. ${ }^{111,112)}$ However, graphene growth at low temperatures using $\mathrm{PAHs}$ results in numerous quality-related defects, making it difficult to be integrated into electronic devices and other applications. ${ }^{113)}$ To address this issue, Lee et $a{ }^{114)}{ }^{114}$ proposed a novel approach that allows low-temperature graphene growth and reduces number of defects. They used a mixture of PAHs with aliphatic carbon sources instead of PAHs alone as the precursors to grow graphene at low temperatures. The aliphatic carbon neutralized the defects related to the native graphene structure by introducing small carbon fragments on the surface of the substrate. As the result, the graphene structures formed defectfree monolayers.

PAHs and 1-octylphosphonic acid (OPA) were added to the substrate and graphene growth was conducted in a CVD system at $400^{\circ} \mathrm{C}$ under a pressure of 0.15 Torr for $90 \mathrm{~min}$. 
(a)

(b)

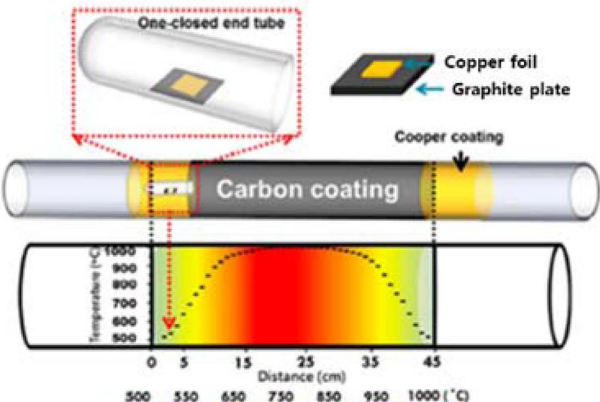

$300 \quad 550 \quad 450 \quad 750 \quad 350 \quad 950 \quad 10001^{\circ} 0$

(c) Front side of Cu foil at $500 \mathrm{C}$

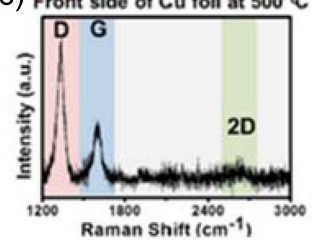

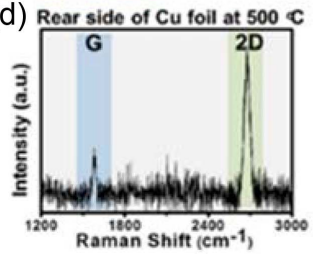

(e)

(f)
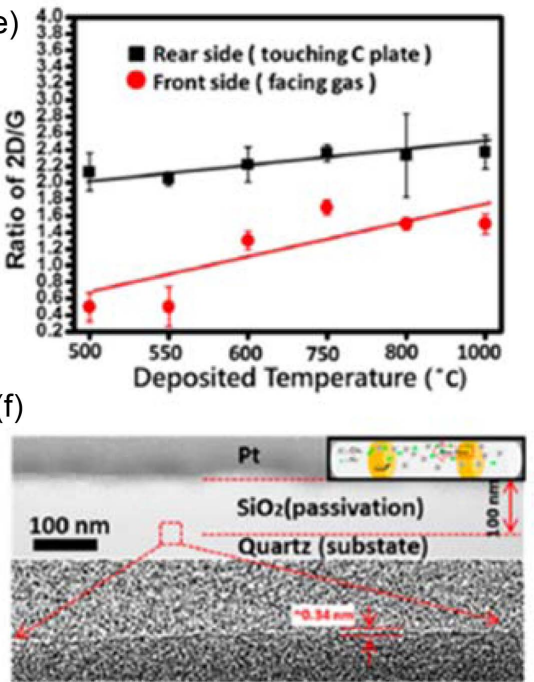

Fig. 19. (a) Experimental setup for CE-CVD graphene growth, (b) temperature distribution in the furnace at a set point of $1000^{\circ} \mathrm{C}$, Raman spectra of graphene grown on (c) the front side of $\mathrm{Cu}$ foil and (d) the rear side of $\mathrm{Cu}$ foil at $\sim 500^{\circ} \mathrm{C}$, (e) intensity ratio of $\mathrm{I}_{2 \mathrm{D}} / \mathrm{I}_{\mathrm{G}}$ as a function of substrate temperature for graphene grown on the front and rear sides of Cu foil, and (f) cross-sectional TEM image of SLG. [Reproduced with permission from ref., ${ }^{110)}$ copyright 2015, American Chemical Society]

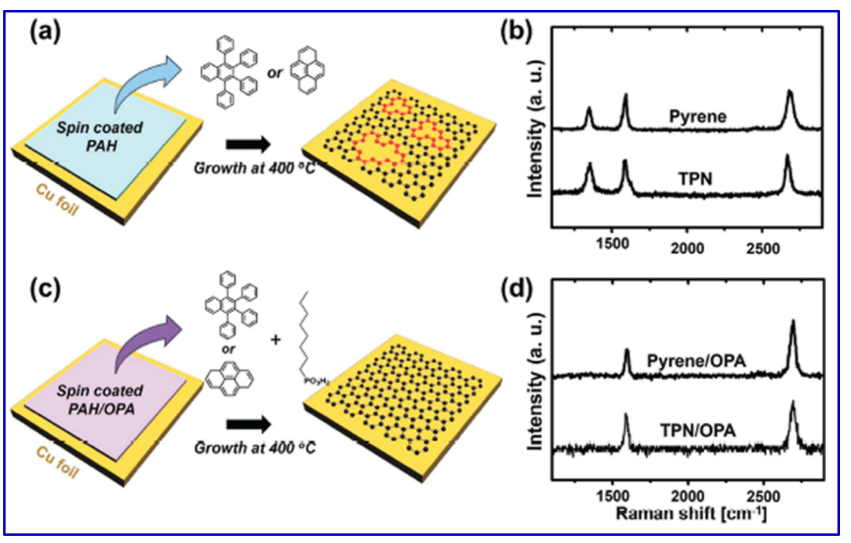

Fig. 20. (a) Molecular structures of 1,2,3,4-tetraphenyl naphthalene (TPN) and pyrene with schematic representations of defective graphene growth on PAH-coated $\mathrm{Cu}$ at low temperature, (b) single Raman spectrum of transferred pyrene- and TPN-derived graphene on $\mathrm{SiO}_{2}$ substrate, (c) schematic representations of high-quality graphene growth on $\mathrm{Cu}$ surface at $400^{\circ} \mathrm{C}$ using $\mathrm{PAH} / \mathrm{OPA}$ heterogeneous solid carbon sources with the molecular structures of pyrene, TPN, and OPA, and (d) single Raman spectrum of transferred pyrene/OPA- and TPN/OPA-derived graphene on $\mathrm{SiO}_{2}$ substrate. [Reproduced with permission from ref., ${ }^{114)}$ copyright 2016, WILEY-VCH Verlag GmbH \& Co. KGaA, Weinheim]

The Raman spectrum of the graphene structure grown using the PAH source (Fig. 20) showed peaks at $1580 \mathrm{~cm}^{-1}$ and $2700 \mathrm{~cm}^{-1}$ with $\mathrm{D}$ and $\mathrm{D}^{\prime}$ peaks at 1350 and $1617 \mathrm{~cm}^{-1}$, respectively. The $I_{D} / I_{D^{\prime}}$ ratio was 4 , indicating that the asgrown graphene structure was composed of vacancy-type defects. On the other hand, the Raman spectra of the other samples showed negligible D peaks, indicating that the graphene grown using the PAH + OPA source consisted of a high-quality monolayer. TEM analysis showed that graphene grown using $\mathrm{PAH}$ alone consisted of a hexagonal array of carbon atoms formed as a monolayer. Notable defects were observed at $1012 \mathrm{~cm}^{-1}$. However, as shown in Fig. 21, the structure grown with OPA alone showed amorphous carbon fragments. These were mainly caused by the conversion reaction of $\mathrm{PAH}$-derived carbon fragments rather than OPA-derived carbon fragments. On the other hand, the structure grown under an optimized OPA-doped PAH source represented a six-membered carbon hexagonal ring, and the grown structure was high-quality SLG. The FET study of both structures also showed that the structure grown with the PAH + OPA source exhibited significantly higher hole and electron mobilities of 1000 and 800 $\mathrm{cm}^{2} \mathrm{~V}^{-1} \mathrm{~s}^{-1}$, respectively. Thus, these results demonstrated that the quality of the as-grown graphene structure using the PAH + OPA source was sufficient for use in electronic devices.

Graphene is considered to have excellent anti-oxidation and -corrosion properties because of its chemically inert nature, resulting in graphene-coated objects being protected from corrosion even in an extremely harsh environment. Zhu's group ${ }^{115)}$ used multi-heating-zone CVD to synthesize graphene on stainless steel substrates from a solid carbon source (Fig. 22). Prior to graphene growth, the stainless steel substrate was annealed at $700^{\circ} \mathrm{C}$ for 5 min under a constant $\mathrm{H}_{2}$ flow of $200 \mathrm{sccm}$. The graphene growth was performed after annealing at a temperature of $500^{\circ} \mathrm{C}$ under $\mathrm{H}_{2}$ and $\mathrm{Ar}$ atmospheres at a total pressure of $2 \mathrm{mTorr}$. The Raman spectra of these graphene structures showed the presence of BLG to FLG. However, graphene structures grown at high $\mathrm{H}_{2}$ flow rates, i.e., > $40 \mathrm{sccm}$, consisted of SLG 

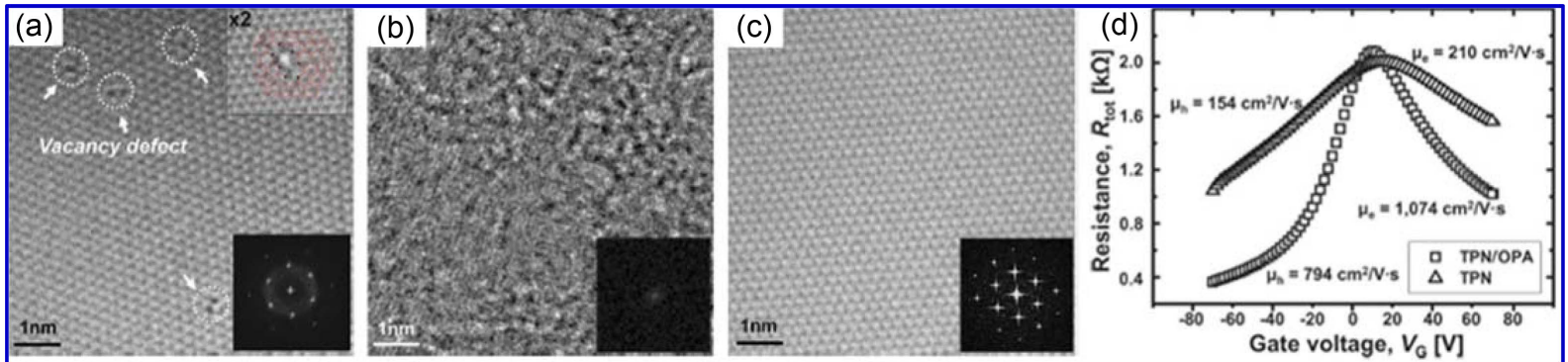

Fig. 21. High-resolution TEM images of graphene samples: (a) TPN-derived graphene, (b) OPA-derived graphene, and (c) TPN/ OPA-derived graphene at $400^{\circ} \mathrm{C}$ (white arrows: point defects. Inset: fast Fourier transforms) and (d) resistance of graphene samples as a function of gate voltage. [Reproduced with permission from ref., ${ }^{113)}$ copyright 2016, WILEY-VCH Verlag GmbH \& Co. KGaA, Weinheim]

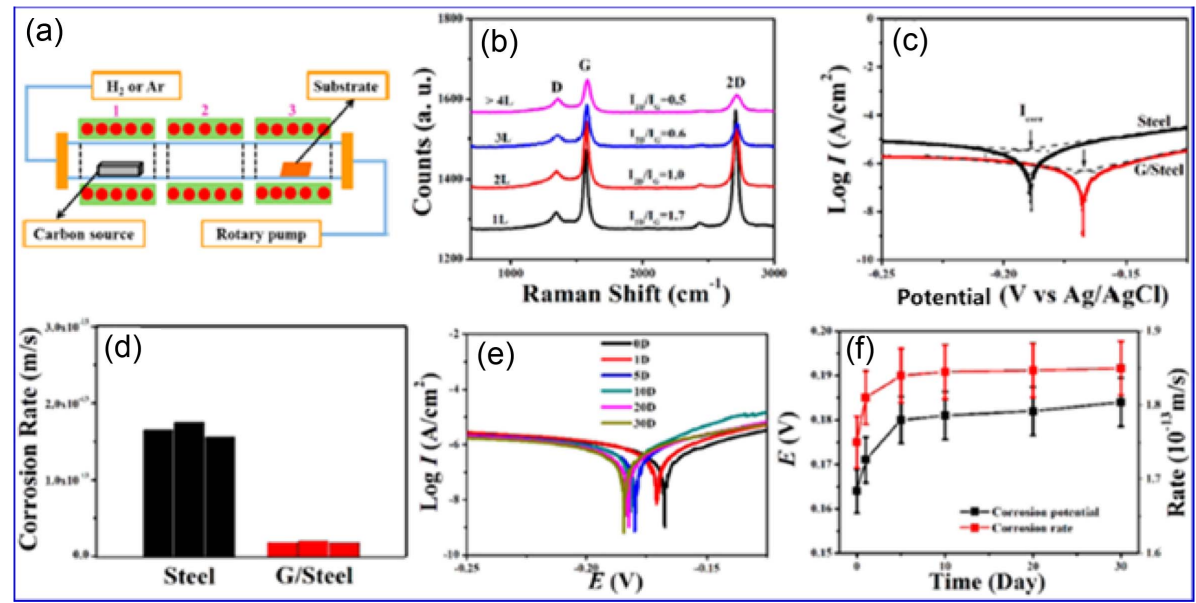

Fig. 22. (a) CVD growth on target substrates using solid carbon source, (b) Raman spectra of PS-derived graphene samples with controllable layer numbers at $500^{\circ} \mathrm{C}$ by varying gas flow rate, (c) Tafel plots of steel and graphene/steel samples in $5 \%$ sea salt solution, (d) corrosion rates of stainless steel and graphene/steel samples extracted from Tafel plots of three different samples, (e) dependence of current-voltage curve vs. time, and (f) corrosion potential and corrosion rate as a function of time for graphene/steel sample. [Reproduced with permission from ref., ${ }^{115)}$ copyright 2016, American Chemical Society]

only. The stainless steel sheets covered by the graphene structures under optimal growth conditions were used as the electrode for the quantitative analysis of corrosion rates using Tafel analysis. The cyclic voltammetry (CV) curves of stainless steel coated with graphene shifted to lower potentials and the current decreased significantly compared to that of bare steel. The corrosion rates for the graphenecoated and bare stainless steel sheets were $2.02 \times 10^{-14}$ and $1.75 \times 10^{-13} \mathrm{~ms}^{-1}$, respectively. These results emphasized the significant anti-corrosion ability of graphene-coated steel electrodes despite being grown at much lower temperatures. The corrosion rate slowly became stable with time on immersing the electrodes in a sea salt solution for 30 days. Quantitative and qualitative studies showed that stainless steel coated with graphene layers had up to 10 times lower corrosion rates than their bare counterparts.

The importance of the structure of the precursor and the intermediate reactions during graphene growth on metal substrates was demonstrated ${ }^{116)}$ by the examination of solid aromatic precursor molecules (anthracene and terphenyl).
All experiments were conducted in a CVD system using copper foil as the substrate at $400^{\circ} \mathrm{C}$ for 20 min under a constant pressure of $4.5 \times 10^{-6}$ Torr. The Raman spectra of graphene grown by $\mathrm{m}$ - and p-terphenyl (m3ph and p2ph, respectively) are shown in Fig. 23. A narrow $2 \mathrm{D}$ peak with a FWHM value of $26 \mathrm{~cm}^{-1}$ and an $\mathrm{I}_{2 \mathrm{D}} / \mathrm{I}_{\mathrm{G}}$ ratio of $\sim 2.5$ was observed, which clearly indicated that the graphene structures grown using $\mathrm{m} 3 \mathrm{ph}$ and $\mathrm{p}$-terphenyl precursors were single-layered. Surface topography studies revealed that the growth over a copper surface led to almost complete graphene coverage. A highly regular hexagonal structure was observed by scanning tunneling microscopy (STM) analysis. The growth mechanism of graphene could be broken down to the below steps based on the abovementioned results. Initially, the $\mathrm{m} 3 \mathrm{ph}$ or $\mathrm{p}$-terphenyl precursor molecules were adsorbed on the surface of the copper foil with a specific adsorption energy because of London dispersion forces, ${ }^{111)}$ which greatly influenced the geometry of the adsorbent molecules. These dispersion forces favored molecules with a tilted configuration when under equilibrium of 


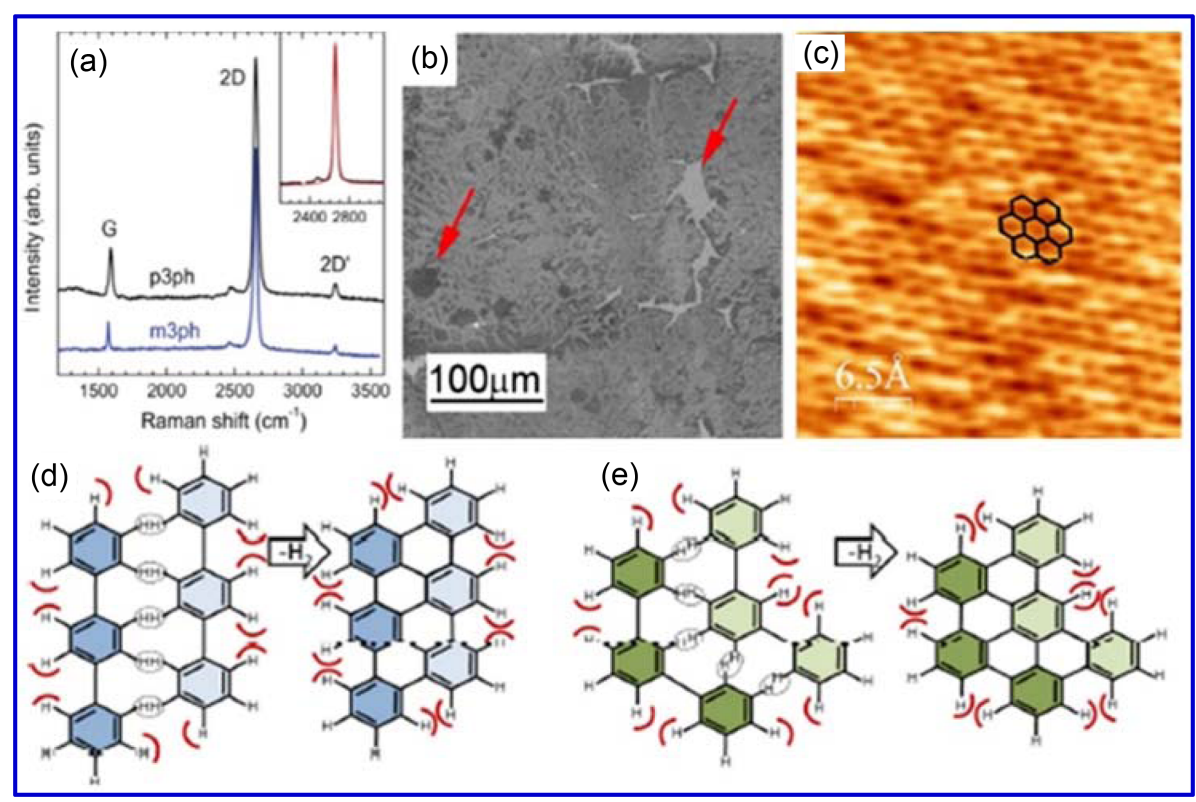

Fig. 23. (a) Raman spectra of graphene grown from p-terphenyl (p2ph, black line) and m-terphenyl (m3ph, blue line) (inset shows 2D band with Lorentzian fit), (b) SEM image of graphene layer grown from p-terphenyl on copper, (c) STM image of graphene layer grown from p-terphenyl (red brackets represent $\mathrm{H}-\mathrm{H}$ repulsion), and sketch of two (d) p-terphenyl and (e) m-terphenyl molecules and the resulting dimer after intermolecular dehydrogenation. [Reproduced with permission from ref., ${ }^{116)}$ copyright 2016, American Chemical Society]

repulsive forces between $\mathrm{C}-\mathrm{H}$ bonds. The active sites from the $\mathrm{C}-\mathrm{H}$ bonds polymerized with adjacent molecules to enable further $\mathrm{C}-\mathrm{C}$ fusions. These newly formed extended aromatic systems with stronger dispersion forces, i.e., polymerization, decreased the total energy of the system and the inter-molecular coalescence continued, ultimately leading to the formation of a graphene structure. The hydrogen atoms linked with the aromatic $\mathrm{C}-\mathrm{H}$ structure in this system remained as active sites for the subsequent attachment of other molecules. ${ }^{117)}$ These results demonstrate and emphasize that precursor geometry and molecular structures are key requirements for graphene growth.

\subsection{Adoption of gaseous precursors}

Graphene growth on single-crystal nickel(111) substrates using ethylene as the precursor via CVD at a partial pressure of $10^{-6}$ Torr was realized. ${ }^{118)}$ Based on in situ microscopy studies, it was evident that the structures grown at temperatures below $650^{\circ} \mathrm{C}$ exhibited thermal stability, whereas those above this temperature exhibited deformation. This temperature was identified as the upper limit for graphene growth over nickel surfaces. Therefore, the selflimiting monolayer growth of graphene on nickel surfaces could be realized by maintaining the growth temperature below $650^{\circ} \mathrm{C}$. Apart from uniformity and monolayer growth over a large-area nickel surface, the presence of nickel carbide along with graphene was especially obvious at temperatures below $460^{\circ} \mathrm{C}$. This was probably attributed to the self-reconstruction(s) of the nickel surface upon adsorption of carbon according to the in-plane conversion mecha- nism. ${ }^{119)}$ As a result, a significant rotation $\left(\sim 3^{\circ}\right)$ between graphene and the underlying nickel surface was observed. ${ }^{120)}$ To explore these issues, Patera et al. ${ }^{121)}$ studied graphene growth over nickel(111) surfaces by in situ STM and X-ray photoelectron spectroscopy. The growth was conducted at temperatures of $400-600^{\circ} \mathrm{C}$ by exposing ethylene $\left(\mathrm{C}_{2} \mathrm{H}_{4}\right)$ at a pressure of $7.5 \times 10^{-5}$ Torr in an ultrahigh vacuum system. The results showed that two key atomistic mechanisms occurred on the $\mathrm{Ni}(111)$ surface when it was exposed to hydrocarbons at around $500^{\circ} \mathrm{C}$. Below $500^{\circ} \mathrm{C}$, conversion of two different nickel carbides led to graphene growth, whereas above $500^{\circ} \mathrm{C}$, graphene predominantly grew directly on the $\mathrm{Ni}(111)$ surface through replacement mechanisms, leading to embedded epitaxial growth along with rotated graphene domains, as shown in Fig. 24.

Growth of high-quality curved graphene structures on nickel templates at a growth temperature of $650^{\circ} \mathrm{C}$ using thermal CVD was realized. ${ }^{122)}$ In a typical process, a nickelcontaining crucible was heated in a hot-wall tube furnace to $650^{\circ} \mathrm{C}$ under $\mathrm{Ar}$ and $\mathrm{H}_{2}$ flow rates of 300 and $30 \mathrm{sccm}$, respectively, for $20 \mathrm{~min}$. Then, $5 \mathrm{sccm}$ of methane gas was introduced for $10 \mathrm{~min}$, and the system was cooled down to room temperature. A schematic demonstration of the growth of curved graphene structures using CVD is shown in Fig. 25. At a desired temperature, methane decomposed over the nickel particles as carbon atoms and dissolved into nickel particles. Upon cooling, the carbon atoms precipitated over the nickel particles and formed curved graphene. Fig. 25 also shows the TEM images of the curved graphene structures grown over a nickel template at $650^{\circ} \mathrm{C}$. These 


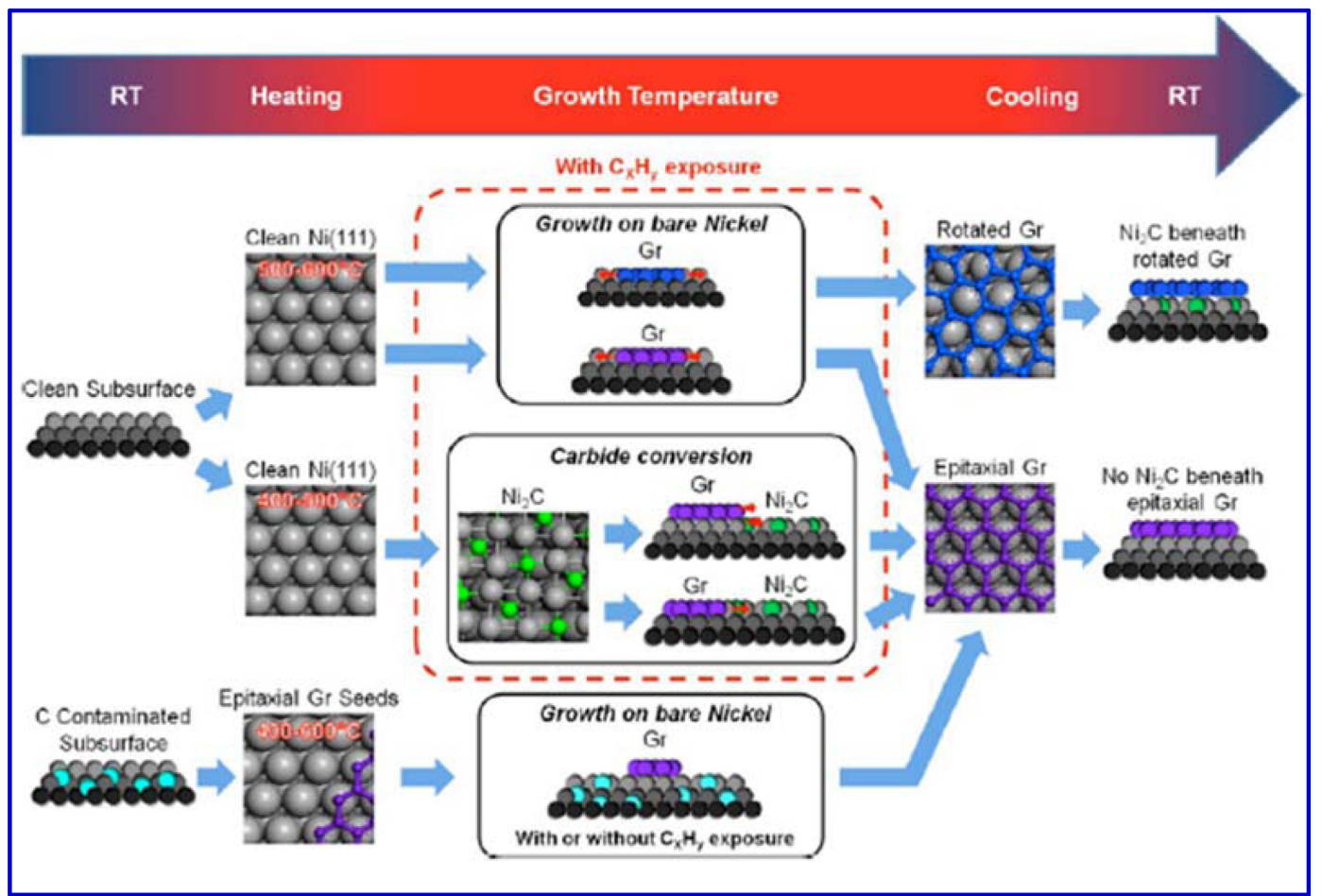

Fig. 24. Overview of different graphene growth routes on $\mathrm{Ni}(111)$. [Reproduced with permission from ref., ${ }^{121)}$ copyright 2013 , American Chemical Society]

results revealed that the as-grown graphene consisted of few-layered or multilayered structures. Electrochemical studies of the curved graphene structures, measured in 3 molL ${ }^{-1} \mathrm{KOH}$ aqueous solution, showed a quasi-rectangular CV profile without any redox peaks. It indicated that the graphene structures consisted of very few surface functional groups. The quasi-triangular charge-discharge curves indicated electrical double-layer electrode characteristics. The specific capacitance of the curved graphene structure was found to be $203 \mathrm{Fg}^{-1}$ at a current density of $1.63 \mathrm{Ag}^{-1}$. Linear increase in the discharge current through the electrodes with an increase in the scan rate indicated efficient charge transport and ion diffusion in the electrodes, which were attributed to the high conductivity and excellent electrochemical properties of the curved graphene structures.

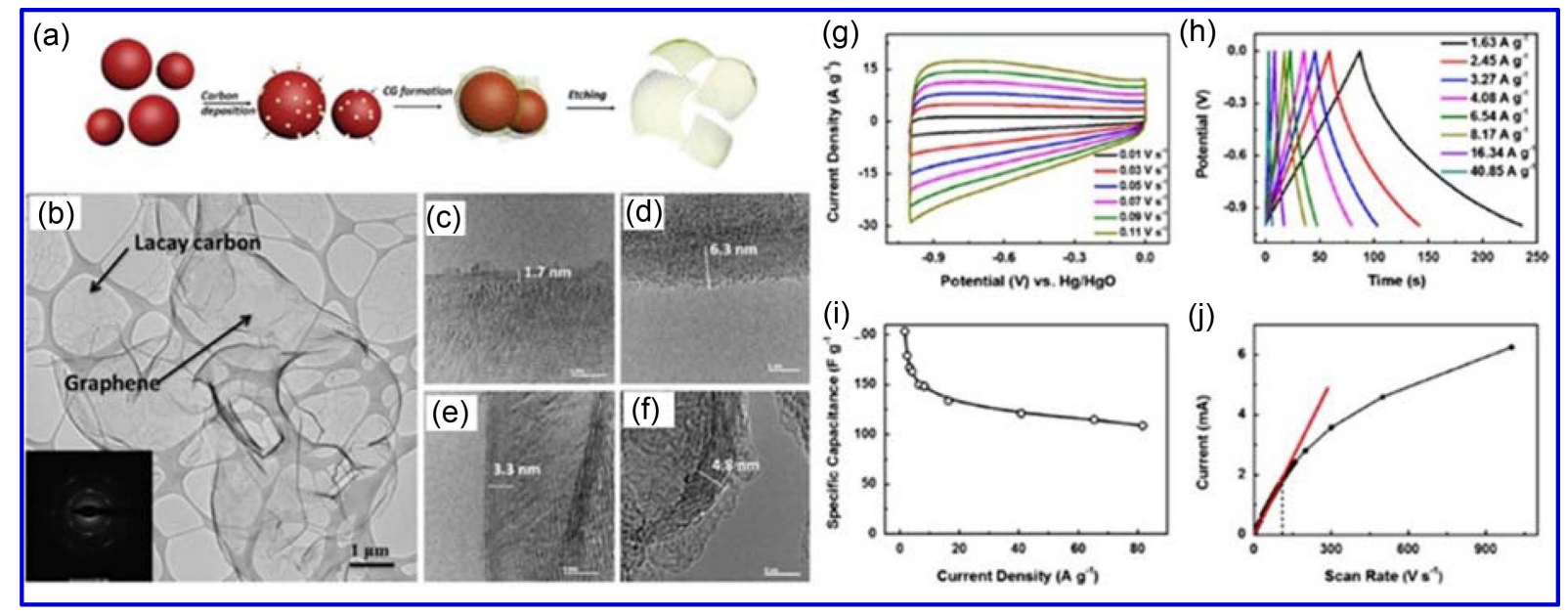

Fig. 25. (a) Growth of curved graphene on Ni particles, (b) TEM image of curved graphene suspended on Lacey carbon (inset shows the selected area electron diffraction (SAED) pattern, (c)-(f) high-resolution TEM images and (g) CV profiles of curved graphene at different scan rates, (h) corresponding galvanostatic charge-discharge curves at different current densities, (i) specific capacitance of curved graphene, and (j) evolution of discharge currents versus different scan rates. [Reproduced with permission from ref., ${ }^{22)}$ copyright 2015, Elsevier] 


\section{Summary and Future Challenges}

A brief review of the first direct growth of graphene on any dielectric flexible and rigid substrate through CVD with or without metal and polymer catalysts is presented in the first part of this study. By making direct graphene growth possible on the target substrate, research on the optimal growth conditions and growth mechanisms in current CVD systems is being conducted. In addition, direct growth of graphene has made it possible to apply graphene in the fields of CMOS technology, energy devices, pressure sensors, transparent conductors, bio-devices and wearable devices. It is necessary to study the correlation between graphene layer number and graphene quality, which is considered to be an existing challenge for direct graphene growth. In fact, the number of graphene layers can be finely controlled and should be selected with reference to the thickness variation and growth time of the metal catalyst. This is essentially a much better demonstration of direct graphene growth on dielectric substrates. The prospect for direct graphene growth has received considerable consideration from the 2D material research community as the "most important issue" when developing new transfer-free graphene for direct manufacturing. Various heterostructured devices are applicable using this direct growth technique.

The challenge of direct growth of graphene is low quality. In addition, for industrial applications, the current technology of CVD systems is not large-scale and limited to small area, but current systems for direct growth of graphene are difficult to apply industrially as the quality of directly grown graphene is inferior to that of graphene obtained by conventional indirect growth. An enormous breakthrough in ultra-modern technology and an accurate understanding of the growth mechanisms of graphene synthesis systems are essential. To achieve this, studies on the growth mechanism and layer number control of graphene have been actively conducted. ${ }^{81,84)}$ The results obtained today have not yet met expectations and require more effort from the $2 \mathrm{D}$ material community. This aim represents an extreme desire as a "secret issue" to achieve the ultimate goal of a large, defectfree graphene-based ultra-low-power electronic device that can be commercialized over the next 20 years.

The second part of the article explores recent low-temperature graphene growth techniques. Significant efforts have been made to grow a continuous layer of high-quality graphene over a large area. For low pressure CVD (LPCVD), high-quality graphene can be obtained by altering the carbon precursor (solid, liquid, and gas sources depending on the decomposition temperature) or metal catalysts $(\mathrm{Cu}, \mathrm{Ni}$, $\mathrm{CuNi})$ for low-temperature growth $\left(\sim 500^{\circ} \mathrm{C}\right)$. In the case of PECVD, the reduced temperature for growing SLG is about $450^{\circ} \mathrm{C}$, which is obtained by changing the plasma type and plasma power used. Due to the formation of faults during low-temperature graphene growth, the overall results of device grade quality and desired size are very poor. Also, there has been little research on the direct growth of a graphene layer on a desired substrate. Moreover, application-based investigation on graphene monolayers conducted so far has been scarce. This is due to the lack of a methodology for growing SLG of the required quality and size directly on a non-catalytic substrate at low temperatures. However, intensive efforts are underway to demonstrate innovative solutions to existing obstacles, and the future generation can undoubtedly benefit from this amazing material.

The low-temperature growth of graphene greatly reduces the production cost and facilitates the development of various devices. However, the main shortcomings of low-temperature growth are the quality, uniformity, crystallinity, and size of the obtained product. It is very difficult to maintain good properties when the graphene structure grows at low temperatures. It is also important to obtain a single layer with growth homogeneity over a large area. Designing a new methodology to overcome this problem of the development of graphene faults is very difficult. In addition, a detailed understanding of the dynamics of low-temperature growth is required, as well as interface and device integration with other materials.

Graphene is a promising material, but its adoption in device manufacturing is still in its nascent stage. An important issue to solve is that it cannot be grown directly on high-resistance and non-catalytic materials. With advances in low-temperature graphene growth and direct growth on the desired substrate/material, efficient adoption of low temperature or directly grown graphene for device integration is still not very efficient and additional developments are required to utilize full potential of graphene on arbitrary substrates. In addition, the transfer of graphene onto a desired substrate requires a transfer process, which leads to defect formation and other problems. For nanoelectronic devices, the graphene transfer process is more complicated and prone to defects. Although there have been some reports on the direct growth of graphene, the growth temperature is still high, which is another major obstacle for graphene integration in nanoelectronics. Therefore, the direct growth of graphene at low temperatures is highly desirable. Thus, multidisciplinary techniques should be researched with a focus on developing methodologies to grow device-quality graphene structures at significantly lower temperatures. To date, graphene growth and lowtemperature growth have been actively studied. However, to further increase the applicability to future graphene devices, it is necessary to identify a technique for directly growing graphene at lower temperatures. Graphene lowtemperature/direct growth technology is expected to realize the application of graphene in various industries and to expand the commercial application fields of graphene. 


\section{REFERENCES}

1. H. Kim, A. A. Abdala, and C. W. Macosko, "Graphene/ Polymer Nanocomposites," Macromolecules, 43 [16] 651530 (2010)

2. R. J. Young, I. A. Kinloch, L. Gong, and K. S. Novoselov, "The Mechanics of Graphene nanocomposites: A Review," Compos. Sci. Technol., 72 [12] 1459-76 (2012).

3. J. S. Bunch, A. M. van der Zande, S. S. Verbridge, I. W. Frank, D. M. Tanenbaum, J. M. Parpia, H. G. Craighead, and P. L. McEuen, "Electromechanical Resonators from Graphene Sheets," Science, 315 [5811] 490-93 (2007).

4. M. J. Allen, V. C. Tung, and R. B. Kaner, "Honeycomb Carbon: A Review of Graphene," Chem. Rev., 110 [1] 13245 (2010).

5. A. H. Castro Neto, F. Guinea, N. M. R. Peres, K. S. Novoselov, and A. K. Geim, "The Electronic Properties of Graphene," Rev. Mod. Phys., 81 109-62 (2009).

6. F. Schwierz, "Graphene Transistors," Nat. Nanotechnol., 5 487-96 (2010).

7. F. Bonaccorso, Z. Sun, T. Hasan, and A. C. Ferrari, "Graphene Photonics and Optoelectronics," Nat. Photonics, 4 611-22 (2010).

8. D. A. C. Brownson, D. K. Kampouris, and C. E. Banks, "An Overview of Graphene in Energy Production and Storage Applications," J. Power Sources, 196 [11] 4873-85 (2011).

9. S. Park and R. S. Ruoff, "Chemical Methods for the Production of Graphenes," Nat. Nanotechnol., 4 [4] 217-24 (2009).

10. S. P. Pang, J. M. Englert, H. N. Tsao, Y. Hernandez, A. Hirsch, X. L. Feng, and K. Mullen, "Extrinsic Corrugation-Assisted Mechanical Exfoliation of Monolayer Graphene," Adv. Mater., 22 [47] 5374-77 (2010).

11. T. L. Yoon, T. L. Lim, T. K. Min, S. H. Hung, N. Jakse, and S. K. Lai, "Epitaxial Growth of Graphene on 6H-Silicon Carbide Substrate by Simulated Annealing Method," J. Chem. Phys., 139 [20] 204702 (2013).

12. S. W. Poon, W. Chen, E. S. Tok, and A. T. S. Wee, "Probing Epitaxial Growth of Graphene on Silicon Carbide by Metal Decoration," Appl. Phys. Lett., 92 [10] 104102 (2008).

13. W.S. Kim, S. Y. Moon, N. H. Park, H. Huh, K. B. Shim, and H. Ham, "Electrical and Structural Feature of Monolayer Graphene Produced by Pulse Current Unzipping and Microwave Exfoliation of Carbon Nanotubes," Chem. Mater., 23 [4] 940-44 (2011).

14. A. Sinitskii, A. A. Fursina, D. V. Kosynkin, A. L. Higginbotham, D. Natelson, and J. M. Tour, "Electronic Transport in Monolayer Graphene Nanoribbons Produced by Chemical Unzipping of Carbon Nanotubes," Appl. Phys. Lett., 95 [25] 253108 (2009).

15. T. Ciuk, P. Caban, and W. Strupinski, "Charge Carrier Concentration and Offset Voltage in Quasi-Free-Standing Monolayer Chemical Vapor Deposition Graphene on SiC," Carbon, 101 431-38 (2016).

16. M. S. Rosmi, S. M. Shinde, N. D. A. Rahman, A. Thangaraja, S. Sharma, K. P. Sharma, Y. Yaakob, R. K. Vishwakarma, S. A. Bakar, G. Kalita, H. Ohtani, and M. Tanemura, "Synthesis of Uniform Monolayer Graphene on Re-Solidified Copper from Waste Chicken Fat by Low Pressure Chemical Vapor Deposition," Mater. Res. Bull., 83 573-80 (2016).

17. J. W. Suk, W. H. Lee, T. J. Kang, and R. D. Piner, "Transfer of Chemical Vapor Deposition-Grown Monolayer Graphene by Alkane Hydrocarbon," Sci. Adv. Mater., 8 [1] 144-47 (2016).

18. H. J. Tan, Y. Fan, Y. M. Rong, B. Porter, C. S. Lau, Y. Q. Zhou, Z. Y. He, S. S. Wang, H. Bhaskaran, and J. H. Warner, "Doping Graphene Transistors Using Vertical Stacked Monolayer $\mathrm{WS}_{2}$ Heterostructures Grown by Chemical Vapor Deposition," ACS Appl. Mater. Interfaces, 8 [3] 1644-52 (2016).

19. C. Zhao, B. Deng, G. C. Chen, B. Lei, H. Hua, H. L. Peng, and Z. M. Yan, "Large-Area Chemical Vapor DepositionGrown Monolayer Graphene-Wrapped Silver Nanowires for Broad-Spectrum and Robust Antimicrobial Coating," Nano Res., 9 [4] 963-73 (2016).

20. C. C. Chen, C. H. Yeh, C. C. Chang, and J. J. Ho, "Conversion of $\mathrm{CO}_{2}$ and $\mathrm{C}_{2} \mathrm{H}_{6}$ to Propanoic Acid on an IridiumModified Graphene Oxide Surface: Quantum-Chemical Investigation," Ind. Eng. Chem. Res., 54 [5] 1539-46 (2015).

21. Z. Y. Jiang and L. F. Yan, "Conversion of Glucose to Valuable Platform Chemicals over Graphene Solid Acid Catalyst," Chin. J. Chem. Phys., 28 [2] 230-34 (2015).

22. B. S. Shen, J. J. Ding, X. B. Yan, W. J. Feng, J. Li, and Q. J. Xue, "Influence of Different Buffer Gases on Synthesis of Few-Layered Graphene by Arc Discharge Method," Appl. Surf. Sci., 258 [10] 4523-31 (2012).

23. Y. P. Wu, B. Wang, Y. F. Ma, Y. Huang, N. Li, F. Zhang, and Y. S. Chen, "Efficient and Large-Scale Synthesis of Few-Layered Graphene Using an Arc-Discharge Method and Conductivity Studies of the Resulting Films," Nano Res., 3 [9] 661-69 (2010).

24. N. Li, Z. Y. Wang, K. K. Zhao, Z. J. Shi, Z. N. Gu, and S. K. $\mathrm{Xu}$, "Large Scale Synthesis of N-Doped Multi-Layered Graphene Sheets by Simple Arc-Discharge Method," Carbon, 48 [1] 255-59 (2010).

25. Y. X. Zhang, X. He, G. M. Zeng, T. Chen, Z. Y. Zhou, H. T. Wang, and W. J. Lu, "Enhanced Photodegradation of Pentachlorophenol by Single and Mixed Nonionic and Anionic Surfactants Using Graphene- $\mathrm{TiO}_{2}$ as Catalyst," Environ. Sci. Pollut. Res. Int., 22 [22] 18211-20 (2015).

26. A. K. Geim and K. S. Novoselov, "The Rise of Graphene," Nat. Mater., 6 183-91 (2007).

27. C.-Y. Su, A.-Y. Lu, Y. Xu, F.-R. Chen, A. N. Khlobystov, and L.-J. Li, "High-Quality Thin Graphene Films from Fast Electrochemical Exfoliation," ACS Nano, 5 [3] 233239 (2011).

28. Y. Hernandez, V. Nicolosi, M. Lotya, F. M. Blighe, Z. Sun, S. De, I. McGovern, B. Holland, M. Byrne, Y. K. Gun'Ko, J. J. Boland, P. Niraj, G. Duesberg, S. Krishnamurthy, R. Goodhue, J. Hutchison, V. Scardaci, A. C. Ferrari, and J. N. Coleman, "High-Yield Production of Graphene by Liquid-Phase Exfoliation of Graphite," Nat. Nano., 3 563-68 (2008).

29. P. W. Sutter, J.-I. Flege, and E. A. Sutter, "Epitaxial Graphene on Ruthenium," Nat. Mater., 7 406-11 (2008). 
30. C. Berger, Z. Song, X. Li, X. Wu, N. Brown, C. Naud, D. Mayou, T. Li, J. Hass, and A. N. Marchenkov, "Electronic Confinement and Coherence in Patterned Epitaxial Graphene," Science, 312 [5777] 1191-96 (2006).

31. K. V. Emtsev, F. Speck, T. Seyller, L. Ley, and J. D. Riley, "Interaction, Growth, and Ordering of Epitaxial Graphene on SiC $\{0001\}$ Surfaces: A Comparative Photoelectron Spectroscopy Study," Phys. Rev. B, 77155303 (2008).

32. J. Hass, W. A. D. Heer, and E. H. Conrad, "The Growth and Morphology of Epitaxial Multilayer Graphene," J. Phys.: Condens. Matter, 20323202 (2008).

33. C.-Y. Su, Y. Xu, W. Zhang, J. Zhao, A. Liu, X. Tang, C.-H. Tsai, Y. Huang, and L.-J. Li, "Highly Efficient Restoration of Graphitic Structure in Graphene Oxide Using Alcohol Vapors," ACS Nano, 4 [9] 5285-92 (2010).

34. G. Williams, B. Seger, and P. V. Kamat, " $\mathrm{TiO}_{2}$-Graphene Nanocomposites. UV-Assisted Photocatalytic Reduction of Graphene Oxide," ACS Nano, 2 [7] 1487-91 (2008).

35. A. A. Green and M. C. Hersam, "Solution Phase Production of Graphene with Controlled Thickness via Density Differentiation," Nano Lett., 9 [12] 4031-36 (2009).

36. L. J. Cote, F. Kim, and J. Huang, "Langmuir-Blodgett Assembly of Graphite Oxide Single Layers," J. Am. Chem. Soc., 131 [3] 1043-49 (2008).

37. D. Li, M. B. Müller, S. Gilje, R. B. Kaner, and G. G. Wallace, "Processable Aqueous Dispersions of Graphene Nanosheets," Nat. Nanotechnol., 3 101-5 (2008).

38. W. Gao, L.B. Alemany, L. Ci, and P. M. Ajayan, "New Insights into the Structure and Reduction of Graphite Oxide," Nat. Chem., 1 403-8 (2009).

39. K. S. Kim, Y. Zhao, H. Jang, S. Y. Lee, J. M. Kim, K. S. Kim, J.-H. Ahn, P. Kim, J.-Y. Choi, and B. H. Hong, "Large-Scale Pattern Growth of Graphene Films for Stretchable Transparent Electrodes," Nature, 457 706-10 (2009).

40. J. Coraux, A.T. N 'Diaye, C. Busse, and T. Michely, "Structural Coherency of Graphene on $\operatorname{Ir}(111)$," Nano Lett., 8 [2] 565-70 (2008).

41. S. Bae, H. Kim, Y. Lee, X. F. Xu, J. S. Park, Y. Zheng, J. Balakrishnan, T. Lei, H. R. Kim, Y. I. Song, Y. J. Kim, K. S. Kim, B. Ozyilmaz, J. H. Ahn, B. H. Hong, and S. Iijima, "Roll-to-Roll Production of 30-Inch Graphene Films for Transparent Electrodes," Nat. Nanotechnol., 5 [8] 574-78 (2010).

42. A. Reina, X. Jia, J. Ho, D. Nezich, H. Son, V. Bulovic, M. S. Dresselhaus, and J. Kong, "Large Area, Few-Layer Graphene Films on Arbitrary Substrates by Chemical Vapor Deposition," Nano Lett., 9 [1] 30-5 (2008).

43. X. Li, W. Cai, J. An, S. Kim, J. Nah, D. Yang, R. Piner, A. Velamakanni, I. Jung, and E. Tutuc, "Large-Area Synthesis of High-Quality and Uniform Graphene Films on Copper Foils," Science, 324 [5932] 1312-14 (2009).

44. S. Lee, K. Lee, and Z. Zhong, "Wafer Scale Homogeneous Bilayer Graphene Films by Chemical Vapor Deposition," Nano Lett., 10 [11] $4702-7$ (2010).

45. K. Yan, H. Peng, Y. Zhou, H. Li, and Z. Liu, "Formation of Bilayer Bernal Graphene: Layer-by-Layer Epitaxy via Chemical Vapor Deposition," Nano Lett., 11 [3] 1106-10 (2011).
46. Z. Sun, Z. Yan, J. Yao, E. Beitler, Y. Zhu, and J. M. Tour, "Growth of Graphene from Solid Carbon Sources," Nature, 468 549-52 (2010).

47. K. Kim, J.-Y. Choi, T. Kim, S.-H. Cho, and H.-J. Chung, "A Role for Graphene in Silicon-Based Semiconductor Devices," Nature, 479 338-44 (2011).

48. Z. Yan, Z. Peng, and J. M. Tour, "Chemical Vapor Deposition of Graphene Single Crystals," Acc. Chem. Res., 47 [4] 1327-37 (2014).

49. J. Sun, S. Deng, W. Guo, Z. Zhan, J. Deng, C. Xu, X. Fan, K. Xu, W. Guo, and Y. Huang, "Electrochemical Bubbling Transfer of Graphene Using a Polymer Support with Encapsulated Air Gap as Permeation Stopping Layer," J. Nanomater., 201651 (2016).

50. C. T. Cherian, F. Giustiniano, I. Martin-Fernandez, H. Andersen, J. Balakrishnan, and B. Özyilmaz, "BubbleFree' Electrochemical Delamination of CVD Graphene Films," Small, 11 [2] 189-94 (2015).

51. D. Mafra, T. Ming, and J. Kong, "Facile Graphene Transfer Directly to Target Substrates with a Reusable Metal Catalyst," Nanoscale, 7 [36] 14807-12 (2015).

52. Y. Wang, Y. Zheng, X. Xu, E. Dubuisson, Q. Bao, J. Lu, and K. P. Loh, "Electrochemical Delamination of CVDGrown Graphene Film: toward the Recyclable Use of Copper Catalyst," ACS Nano, 5 [12] 9927-33 (2011).

53. Y. Chen, X. L. Gong, and J. G. Gai, "Progress and Challenges in Transfer of Large-Area Graphene Films," Adv. Sci., 3 [8] 1500343 (2016).

54. H. Terrones, R. Lv, M. Terrones, and M. S. Dresselhaus, "The Role of Defects and Doping in 2D Graphene Sheets and 1D Nanoribbons," Rep. Prog. Phys., 75 [6] 062501 (2012).

55. R. Steingrüber, M. Ferstl, and W. Pilz, "Micro-Optical Elements Fabricated by Electron-Beam Lithography and Dry Etching Technique Using Top Conductive Coatings," Microelectron. Eng., 57 285-89 (2001).

56. T. Jiao, J. Liu, D. Wei, Y. Feng, X. Song, H. Shi, S. Jia, W. Sun, and C. Du, "Composite Transparent Electrode of Graphene Nanowalls and Silver Nanowires on Micropyramidal Si for High-Efficiency Schottky Junction Solar Cells," ACS Appl. Mater. Interfaces, 7 [36] 20179-83 (2015).

57. X. Song, T. Sun, J. Yang, L. Yu, D. Wei, L. Fang, B. Lu, C. $\mathrm{Du}$, and D. Wei, "Direct Growth of Graphene Films on 3D Grating Structural Quartz Substrates for High-Performance Pressure-Sensitive Sensors," ACS Appl. Mater. Interfaces, 8 [26] 16869-75 (2016).

58. S. C. Mannsfeld, B. C. Tee, R. M. Stoltenberg, C. V. H. Chen, S. Barman, B. V. Muir, A. N. Sokolov, C. Reese, and Z. Bao, "Highly Sensitive Flexible Pressure Sensors with Microstructured Rubber Dielectric Layers," Nat. Mater., 9 859-64 (2010).

59. H. Ago, Y. Ogawa, M. Tsuji, S. Mizuno, and H. Hibino, "Catalytic Growth of Graphene: Toward Large-Area Single-Crystalline Graphene," J. Phys. Chem. Lett., 3 [16] 2228-36 (2012).

60. X. S. Li, W. W. Cai, J. H. An, S. Kim, J. Nah, D. X. Yang, R. Piner, A. Velamakanni, I. Jung, E. Tutuc, S. K. Banerjee, L. Colombo, and R. S. Ruoff, "Large-Area Synthesis of 
High-Quality and Uniform Graphene Films on Copper Foils," Science, 324 [5932] 1312-14 (2009).

61. X. S. Li, C. W. Magnuson, A. Venugopal, R. M. Tromp, J. B. Hannon, E. M. Vogel, L. Colombo, and R. S. Ruoff, "Large-Area Graphene Single Crystals Grown by LowPressure Chemical Vapor Deposition of Methane on Copper," J. Am. Chem. Soc., 133 [9] 2816-19 (2011).

62. X. C. Dong, P. Wang, W. J. Fang, C. Y. Su, Y. H. Chen, L. J. Li, W. Huang, and P. Chen, "Growth of Large-Sized Graphene Thin-Films by Liquid Precursor-Based Chemical Vapor Deposition under Atmospheric Pressure," Carbon, 49 [11] 3672-78 (2011).

63. A. Guermoune, T. Chari, F. Popescu, S. S. Sabri, J. Guillemette, H. S. Skulason, T. Szkopek, and M. Siaj, "Chemical Vapor Deposition Synthesis of Graphene on Copper with Methanol, Ethanol, and Propanol Precursors," Carbon, 49 [13] 4204-10 (2011).

64. A. Srivastava, C. Galande, L. Ci, L. Song, C. Rai, D. Jariwala, K. F. Kelly, and P. M. Ajayan, "Novel Liquid Precursor-Based Facile Synthesis of Large-Area Continuous, Single, and Few-Layer Graphene Films," Chem. Mater., 22 [11] 3457-61 (2010).

65. Y. G. Yao, Z. Li, Z. Y. Lin, K. S. Moon, J. Agar, and C. P. Wong, "Controlled Growth of Multilayer, Few-Layer, and Single-Layer Graphene on Metal Substrates," J. Phys. Chem. C, 115 [13] 5232-38 (2011).

66. Z. C. Li, P. Wu, C. X. Wang, X. D. Fan, W. H. Zhang, X. F. Zhai, C. G. Zeng, Z. Y. Li, J. L. Yang, and J. G. Hou, "LowTemperature Growth of Graphene by Chemical Vapor Deposition Using Solid and Liquid Carbon Sources," ACS Nano, 5 [4] 3385-90 (2011).

67. M. H. Rummeli, A. Bachmatiuk, A. Scott, F. Borrnert, J. H. Warner, V. Hoffman, J.-H. Lin, G. Cuniberti, and B. Buchner, "Direct Low-Temperature Nanographene CVD Synthesis over a Dielectric Insulator," ACS Nano, 4 [7] 4206-10 (2010).

68. L. Zhang, Z. Shi, Y. Wang, R. Yang, D. Shi, and G. Zhang, "Catalyst-Free Growth of Nanographene Films on Various Substrates," Nano Res., 4 [3] 315-21 (2011).

69. J. Chen, Y. Wen, Y. Guo, B. Wu, L. Huang, Y. Xue, D. Geng, D. Wang, G. Yu, and Y. Liu, "Oxygen-Aided Synthesis of Polycrystalline Graphene on Silicon Dioxide Substrates," J. Am. Chem. Soc., 133 [4] 17548-51 (2011).

70. H. J. Shin, W. M. Choi, S. M. Yoon, G. H. Han, Y. S. Woo, E. S. Kim, S. J. Chae, X. S. Li, A. Benayad, D. D. Loc, F. Gunes, Y. H. Lee, and J. Y. Choi, "Transfer-Free Growth of Few-Layer Graphene by Self-Assembled Monolayers," Adv. Mater., 23 [38] 4392-97 (2011).

71. M. P. Levendorf, C. S. Ruiz-Vargas, S. Garg, and J. Park, "Transfer-Free Batch Fabrication of Single Layer Graphene Transistors," Nano Lett., 9 [12] 4479-83 (2009).

72. S.-J. Byun, H. Lim, G.-Y. Shin, T.-H. Han, S. H. Oh, J.-H. Ahn, H. C. Choi, and T.-W. Lee, "Graphenes Converted from Polymers," J. Phys. Chem. Lett., 2 [5] 493-97 (2011).

73. C. S. Lee, L. Baraton, Z. He, J.-L. Maurice, M. Chaigneau, D. Pribat, and C. S. Cojocaru, "Dual Graphene films growth process based on plasma-assisted chemical vapor deposition, in: SPIE NanoScience+ Engineering, Interna- tional Society for Optics and Photonics, 2010, pp. 77610P$77610 \mathrm{P}-77617$.

74. X. Li, W. Cai, L. Colombo, and R. S. Ruoff, "Evolution of Graphene Growth on $\mathrm{Ni}$ and $\mathrm{Cu}$ by Carbon Isotope Labeling," Nano Lett., 9 [12] 4268-72 (2009).

75. X. Li, Y. Zhu, W. Cai, M. Borysiak, B. Han, D. Chen, R. D. Piner, L. Colombo, and R. S. Ruoff, "Transfer of LargeArea Graphene Films for High-Performance Transparent Conductive Electrodes," Nano Lett., 9 [12] 4359-63 (2009).

76. H. J. Song, M. Son, C. Park, H. Lim, M. P. Levendorf, A. W. Tsen, J. Park, and H. C. Choi, "Large Scale Metal-Free Synthesis of Graphene on Sapphire and Transfer-Free Device Fabrication," Nanoscale, 4 [10] 3050-54 (2012).

77. J. M. P. Alaboson, Q. H. Wang, J. D. Emery, A. L. Lipson, M. J. Bedzyk, J. W. Elam, M. J. Pellin, and M. C. Hersam, "Seeding Atomic Layer Deposition of High-k Dielectrics on Epitaxial Graphene with Organic Self-Assembled Monolayers," ACS Nano, 5 [6] 5223-32 (2011).

78. P.-Y. Teng, C.-C. Lu, K. Akiyama-Hasegawa, Y.-C. Lin, C.-H. Yeh, K. Suenaga, and P.-W. Chiu, "Remote Catalyzation for Direct Formation of Graphene Layers on Oxides," Nano Lett., 12 [3] 1379-84 (2012).

79. J. Kang, D. Shin, S. Bae, and B. H. Hong, "Graphene Transfer: Key for Applications," Nanoscale, 4 [18] 5527-37 (2012).

80. Y.-J. Kim, S. J. Kim, M. H. Jung, K. Y. Choi, S. Bae, S.-K. Lee, Y. Lee, D. Shin, B. Lee, H. Shin, M. Choi, K. Park, JH Ahn, and B. H. Hong, "Low-Temperature Growth and Direct Transfer of Graphene-Graphitic Carbon Films on Flexible Plastic Substrates," Nanotechnology, 23 [34] 344016 (2012).

81. H.-K. Seo, K. Kim, S.-Y. Min, Y. Lee, C. E. Park, R. Raj, and T.-W. Lee, "Direct Growth of Graphene-Dielectric BiLayer Structure on Device Substrates from Si-Based Polymer," 2D Mater., 4 [2] 024001 (2017).

82. J. Yamada, Y. Ueda, T. Maruyama, and S. Naritsuka, "Direct Growth of Multilayer Graphene by Precipitation Using W Capping Layer,” Jpn. J. Appl. Phys., 55 [10] 100302 (2016).

83. C.-Y. Su, A.-Y. Lu, C.-Y. Wu, Y.-T. Li, K.-K. Liu, W. Zhang, S.-Y. Lin, Z.-Y. Juang, Y.-L. Zhong, and F.-R. Chen, "Direct Formation of Wafer Scale Graphene Thin Layers on Insulating Substrates by Chemical Vapor Deposition," Nano Lett., 11 [9] 3612-16 (2011).

84. Z. Peng, Z. Yan, Z. Sun, and J. M. Tour, "Direct Growth of Bilayer Graphene on $\mathrm{SiO}_{2}$ Substrates by Carbon Diffusion through Nickel," ACS Nano, 5 [10] 8241-47 (2011).

85. M. Min, S. Seo, Y. Yoon, K. Cho, S. M. Lee, T. Lee, and H. Lee, "Catalyst-Free Bottom-Up Growth of Graphene Nanofeatures along with Molecular Templates on Dielectric Substrates," Nanoscale, 8 [38] 17022-29 (2016).

86. Z. Liu, L. Song, S. Zhao, J. Huang, L. Ma, J. Zhang, J. Lou, and P. M. Ajayan, "Direct Growth of Graphene/Hexagonal Boron Nitride Stacked Layers," Nano Lett., 11 [5] 2032-37 (2011).

87. P. Thanh Trung, J. Campos-Delgado, F. Joucken, J.-F. Colomer, B. Hackens, J.-P. Raskin, C. N. Santos, and S. Robert, "Direct Growth of Graphene on Si (111)," J. Appl. Phys., 115 [22] 223704 (2014). 
88. S. C. Xu, B. Y. Man, S. Z. Jiang, C. S. Chen, C. Yang, M. Liu, X. G. Gao, Z. C. Sun, and C. Zhang, "Direct Synthesis of Graphene on $\mathrm{SiO}_{2}$ Substrates by Chemical Vapor Deposition," CrystEngComm, 15 [10] 1840-44 (2013).

89. J. Kwak, J. H. Chu, J.-K. Choi, S.-D. Park, H. Go, S. Y. Kim, K. Park, S.-D. Kim, Y.-W. Kim, and E. Yoon, "Near Room-Temperature Synthesis of Transfer-Free Graphene Films," Nat. Commun., 3645 (2012).

90. H. Bi, S. Sun, F. Huang, X. Xie, and M. Jiang, "Direct Growth of Few-Layer Graphene Films on $\mathrm{SiO}_{2}$ Substrates and Their Photovoltaic Applications," J. Mater. Chem., 22 [2] 411-16 (2012).

91. H. Kim, I. Song, C. Park, M. Son, M. Hong, Y. Kim, J. S. Kim, H.-J. Shin, J. Baik, and H. C. Choi, "Copper-VaporAssisted Chemical Vapor Deposition for High-Quality and Metal-Free Single-Layer Graphene on Amorphous $\mathrm{SiO}_{2}$ Substrate," ACS Nano, 7 [8] 6575-82 (2013).

92. J. Li, C. Shen, Y. Que, Y. Tian, L. Jiang, D. Bao, Y. Wang, S. Du, and H.-J. Gao, "Copper Vapor-Assisted Growth of Hexagonal Graphene Domains on Silica Islands," Appl. Phys. Lett., 109 [2] 023106 (2016).

93. S. Tang, H. Wang, H. S. Wang, Q. Sun, X. Zhang, C. Cong, H. Xie, X. Liu, X. Zhou, and F. Huang, "Silane-Catalysed Fast Growth of Large Single-Crystalline Graphene on Hexagonal Boron Nitride," Nat. Commun., 66499 (2015).

94. G. Yang, H.-Y. Kim, S. Jang, and J. Kim, "Transfer-Free Growth of Multilayer Graphene Using Self-Assembled Monolayers," ACS Appl. Mater. Interfaces, 8 [40] 27115-21 (2016).

95. H. Wang, Y. Zhou, D. Wu, L. Liao, S. L. Zhao, H. L. Peng, and Z. F. Liu, "Synthesis of Boron-Doped Graphene Monolayers Using the Sole Solid Feedstock by Chemical Vapor Deposition," Small, 9 [8] 1316-20 (2013).

96. R. Mehta, S. Chugh, and Z. Chen, "Transfer-Free MultiLayer Graphene as a Diffusion Barrier," Nanoscale, 9 [5] 1827-33 (2017).

97. R. Muñoz, C. Munuera, J. Martínez, J. Azpeitia, C. Gómez-Aleixandre, and M. García-Hernández, "Low Temperature Metal Free Growth of Graphene on Insulating Substrates by Plasma Assisted Chemical Vapor Deposition," 2D Mater., 4015009 (2016).

98. J. Pang, R. G. Mendes, P. S. Wrobel, M. D. Wlodarski, H. Q. Ta, L. Zhao, L. Giebeler, B. Trzebicka, T. Gemming, and L. Fu, "A Self Terminating Confinement Approach for Large Area Uniform Monolayer Graphene Directly over $\mathrm{Si} / \mathrm{SiO}_{\mathrm{x}}$ by Chemical Vapor Deposition," ACS Nano, 11 [2] 1946-56 (2017).

99. J. Sun, Y. Chen, M. K. Priydarshi, Z. Chen, A. Bachmatiuk, Z. Zou, Z. Chen, X. Song, Y. Gao, and M. H. Ruümmeli, "Direct Chemical Vapor Deposition-Derived Graphene Glasses Targeting Wide Ranged Applications," Nano Lett., 15 [9] 5846-54 (2015).

100. J.-H. Lee, M.-S. Kim, J.-Y. Lim, S.-H. Jung, S.-G. Kang, H.-J. Shin, J.-Y. Choi, S.-W. Hwang, and D. Whang, "CMOS-Compatible Catalytic Growth of Graphene on a Silicon Dioxide Substrate," Appl. Phys. Lett., 109 [5] 053102 (2016).

101. A. Ismach, C. Druzgalski, S. Penwell, A. Schwartzberg, M. Zheng, A. Javey, J. Bokor, and Y. Zhang, "Direct
Chemical Vapor Deposition of Graphene on Dielectric Surfaces," Nano Lett., 10 [5] 1542-48 (2010).

102. J. Xue, J. Sanchez-Yamagishi, D. Bulmash, P. Jacquod, A. Deshpande, K. Watanabe, T. Taniguchi, P. Jarillo-Herrero, and B. J. LeRoy, "Scanning Tunnelling Microscopy and Spectroscopy of Ultra-Flat Graphene on Hexagonal Boron Nitride," Nat. Mater., 10 282-85 (2011).

103. T. Q. Trung and N.-E. Lee, "Materials and Devices for Transparent Stretchable Electronics," J. Mater. Chem. C, 5 [9] 2202-22 (2017).

104. T. Q. Trung and N. E. Lee, "Recent Progress on Stretchable Electronic Devices with Intrinsically Stretchable Components," Adv. Mater., 29 [3] 1603167 (2017).

105. M. Son, H. Lim, M. Hong, and H. C. Choi, "Direct Growth of Graphene Pad on Exfoliated Hexagonal Boron Nitride Surface," Nanoscale, 3 [8] 3089-93 (2011).

106. Q. K. Yu, L.A. Jauregui, W. Wu, R. Colby, J. F. Tian, Z. H. Su, H. L. Cao, Z. H. Liu, D. Pandey, D. G. Wei, T. F. Chung, P. Peng, N. P. Guisinger, E. A. Stach, J. M. Bao, S. S. Pei, and Y. P. Chen, "Control and Characterization of Individual Grains and Grain Boundaries in Graphene Grown by Chemical Vapour Deposition," Nat. Mater., 10 443-49 (2011).

107. B. Zhang, W. H. Lee, R. Piner, I. Kholmanov, Y. P. Wu, H. F. Li, H. X. Ji, and R. S. Ruoff, "Low-Temperature Chemical Vapor Deposition Growth of Graphene from Toluene on Electropolished Copper Foils," ACS Nano, 6 [3] 247176 (2012).

108. J. Jang, M. Son, S. Chung, K. Kim, C. Cho, B. H. Lee, and M. H. Ham, "Low-Temperature-Grown Continuous Graphene Films from Benzene by Chemical Vapor Deposition at Ambient Pressure," Sci. Rep., 517955 (2015).

109. L. Jiang, T. C. Niu, X. Q. Lu, H. L. Dong, W. Chen, Y. Q. Liu, W. P. Hu, and D. B. Zhu, "Low-Temperature, BottomUp Synthesis of Graphene via a Radical-Coupling Reaction,” J. Am. Chem. Soc., 135 [24] 9050-54 (2013).

110. Y. Z. Chen, H. Medina, H. W. Tsai, Y. C. Wang, Y. T. Yen, A. Manikandan, and Y. L. Chueh, "Low Temperature Growth of Graphene on Glass by Carbon-Enclosed Chemical Vapor Deposition Process and Its Application as Transparent Electrode," Chem. Mater., 27 [5] 1646-55 (2015).

111. J. H. Choi, Z. C. Li, P. Cui, X. D. Fan, H. Zhang, C. G. Zeng, and Z. Y. Zhang, "Drastic Reduction in the Growth Temperature of Graphene on Copper via Enhanced London Dispersion Force," Sci. Rep., 31925 (2013).

112. J. Zhang, J. J. Li, Z. L. Wang, X. N. Wang, W. Feng, W. Zheng, W. W. Cao, and P. A. Hu, "Low-Temperature Growth of Large-Area Heteroatom-Doped Graphene Film," Chem. Mater., 26 [7] 2460-66 (2014).

113. X. Wan, K. Chen, D. Q. Liu, J. Chen, Q. Miao, and J. B. $\mathrm{Xu}$, "High-Quality Large-Area Graphene from Dehydrogenated Polycyclic Aromatic Hydrocarbons," Chem. Mater., 24 [20] 3906-15 (2012).

114. E. Lee, H. C. Lee, S. B. Jo, H. Lee, N. S. Lee, C. G. Park, S. K. Lee, H. H. Kim, H. Bong, and K. Cho, "Heterogeneous Solid Carbon Source-Assisted Growth of High-Quality Graphene via CVD at Low Temperatures," Adv. Funct. Mater., 26 [4] 562-68 (2016). 
115. M. M. Zhu, Z. H. Du, Z. Y. Yin, W. W. Zhou, Z. D. Liu, S. H. Tsang, and E. H. T. Teo, "Low-Temperature in Situ Growth of Graphene on Metallic Substrates and Its Application in Anticorrosion," ACS Appl. Mater. Interfaces, 8 [1] 502-10 (2016).

116. K. Gharagozloo-Hubmann, N. S. Muller, M. Giersig, C. Lotze, K. J. Franke, and S. Reicht, "Requirement on Aromatic Precursor for Graphene Formation," J. Phys. Chem. C, 120 [18] 9821-25 (2016).

117. M. Marschall, J. Reichert, K. Seufert, W. Auwarter, F. Klappenberger, A. Weber-Bargioni, S. Klyatskaya, G. Zoppellaro, A. Nefedov, T. Strunskus, C. Woll, M. Ruben, and J. V. Barth, "Supramolecular Organization and Chiral Resolution of p-Terphenyl-m-Dicarbonitrile on the Ag(111) Surface," Chemphyschem, 11 [7] 1446-51 (2010).

118. R. Addou, A. Dahal, P. Sutter, and M. Batzill, "Monolayer Graphene Growth on Ni(111) by Low Temperature Chemical Vapor Deposition," Appl. Phys. Lett., 100 [2] 021601 (2012).
119. C. Klink, I. Stensgaard, F. Besenbacher, and E. Laegsgaard, "An Stm Study of Carbon-Induced Structures on $\mathrm{Ni}(111)$ - Evidence for a Carbidic-Phase Clock Reconstruction," Surf. Sci., 342 [1-3] 250-60 (1995).

120. J. Lahiri, T. Miller, L. Adamska, I. I. Oleynik, and M. Batzill, "Graphene Growth on $\mathrm{Ni}(111)$ by Transformation of a Surface Carbide," Nano Lett., 11 [2] 518-22 (2011).

121. L. L. Patera, C. Africh, R. S. Weatherup, R. Blume, S. Bhardwaj, C. Castellarin-Cudia, A. Knop-Gericke, R. Schloegl, G. Comelli, S. Hofmann, and C. Cepek, "In Situ Observations of the Atomistic Mechanisms of Ni Catalyzed Low Temperature Graphene Growth," ACS Nano, 7 [9] 7901-12 (2013).

122. S. Zhou, J. L. Xu, Y. B. Xiao, N. Zhao, and C. P. Wong, "Low-Temperature Ni Particle-Templated Chemical Vapor Deposition Growth of Curved Graphene for Supercapacitor Applications," Nano Energy, 13 458-66 (2015). 